\title{
Can added value be expected in RCM-simulated large scales?
}

\author{
Emilia Paula Diaconescu • René Laprise
}

Received: 21 June 2012/ Accepted: 22 December 2012/Published online: 16 January 2013

(C) The Author(s) 2013. This article is published with open access at Springerlink.com

\begin{abstract}
Nested Limited-Area Models require driving data to define their lateral boundary conditions (LBC). The optimal choice of domain size and the repercussions of LBC errors on Regional Climate Model (RCM) simulations are important issues in dynamical downscaling work. The main objective of this paper is to investigate the effect of domain size, particularly on the larger scales, and to question whether an RCM, when run over very large domains, can actually improve the large scales compared to those of the driving data. This study is performed with a detailed atmospheric model in its global and regional configurations, using the "Imperfect Big-Brother" (IBB) protocol. The ERA-Interim reanalyses and five global simulations are used to drive RCM simulations for five winter seasons, on four domain sizes centred over the North American continent. Three variables are investigated: precipitation, specific humidity and zonal wind component. The results following the IBB protocol show that, when an RCM is driven by perfect LBC, its skill at reproducing the large scales decreases with increasing the domain of integration, but the errors remain small even for very large domains. On the other hand, when driven by LBC that contain errors, RCMs can bring some reduction of errors in large scales when very large domains are used. The improvement is found especially in the amplitude of patterns of both the stationary and the intra-seasonal transient components. When large errors are present in the $\mathrm{LBC}$, however, these are only partly corrected by the RCM.
\end{abstract}

\footnotetext{
E. P. Diaconescu $(\square) \cdot$ R. Laprise

Department of Earth and Atmospheric Sciences, Centre ESCER

(Étude et Simulation du Climat à l'Échelle Régionale),

University of Québec at Montréal (UQAM), Stn. Downtown,

P.O. Box 8888, Montreal, QC H3C 3P8, Canada

e-mail: diacones@sca.uqam.ca
}

Although results showed that an RCM can have some skill at improving imperfect large scales supplied as driving LBC, the main added value of an RCM is provided by its small scales and its skill to simulate extreme events, particularly for precipitation. Under the IBB protocol all RCM simulations were fairly skilful at reproducing small scales statistics, although the skill decreased with increasing LBC errors. Coarse-resolution model simulations have difficulties in simulating heavy precipitation events, and as a result their precipitation distributions are systematically shifted toward smaller intensity. Under the IBB protocol, all RCM simulations have distributions very similar to the reference field, being little affected by LBC errors, and no significant differences were found between the small scales statistics and the precipitation distributions obtained over different RCM domains.

Keywords Regional climate model · Big-Brother experiment $\cdot$ Lateral boundary errors $\cdot$ Scale decomposition $\cdot$ Added value

\section{Introduction}

The climate-change projections need large ensembles of simulations with coupled General Circulation Models (GCMs) that are run over long periods of time, demanding high computational resources. As a consequence, an affordable computational cost forces the use of GCMs with coarse horizontal resolutions varying between $1^{\circ}$ and $5^{\circ}$. Local, fine-scale climate details that are required for most impact studies can be provided by dynamical downscaling with nested limited-area high-resolution Regional Climate Models (RCMs) run over the region of interest, using the GCM coarse-resolution fields as lateral boundary 
Table 1 Horizontal resolution of the BB simulations

\begin{tabular}{llllll}
\hline Simulation name & PBB & IBB1 & IBB2 & IBB3 & IBB4 \\
\hline Horizontal resolution & $0.45^{\circ}$ & $0.9^{\circ}$ & $1.8^{\circ}$ & $2.25^{\circ}$ & $3.6^{\circ}$ \\
\hline
\end{tabular}

conditions (LBC). The better representation of the topography, coastlines and land-surface properties in high-resolution RCM allows the development of small-scale processes. Laprise et al. (2008) and Rummukainen (2010) described the RCM as a "magnifying glass" that reveals fine scales features that are latent, but are not present in the coarse-resolution driving fields. This also means that the small-scales features are closely related to the large scales, and therefore a good representation of the large scales is a prerequisite for well-simulating small-scales features (Diaconescu et al. 2007; Laprise et al. 2012).

Several studies have shown that the RCMs are able to reproduce well the large- and small-scale climate statistics when driven with analysed LBC (e.g. Jones et al. 1995; Christensen et al. 1997; Machenhauer et al. 1998; Frei et al. 2003; Rinke et al. 2006; Jacob et al. 2007), or under the idealised Big-Brother Experiment (e.g. Denis et al. 2002b, 2003; Antic et al. 2004; Dimitrijevic and Laprise 2005). However, in most applications, the LBC are not perfect, especially in the case they come from GCM simulations. Even reanalyses have some degree of error that can have an impact on the RCM simulations. An example is the study of Yang et al. (2011) who showed that simulations of the East Asian summer monsoon forced by three different reanalysis data exhibit large differences; they argued that these differences are primarily caused by the differences existed in the lateral boundary moisture fluxes and question which LBC are better to use. The problem is of course much more serious when driving with GCM-simulated data.

The impact of LBC errors represents an important issue in RCM studies and its study constitutes the focus of several papers (e.g., Rinke and Dethloff 2000; Misra 2007; Diaconescu et al. 2007; Noguer et al. 1998; Køltzow et al. 2008). Two important processes come into play in the evolution of errors related to LBC.

First, the technique of nesting itself may contribute to large-scale errors even when driving an RCM with perfect LBC. Nesting errors may arise from spatial and time interpolations, as well as the specific way in which nesting is applied. One common problem is the appearance of strong gradients along the lateral boundaries, particularly at the downstream of the dominant flow, due to the mismatch between the inner solution of the RCM and the imposed LBC. The problem can be minimized by using relaxation

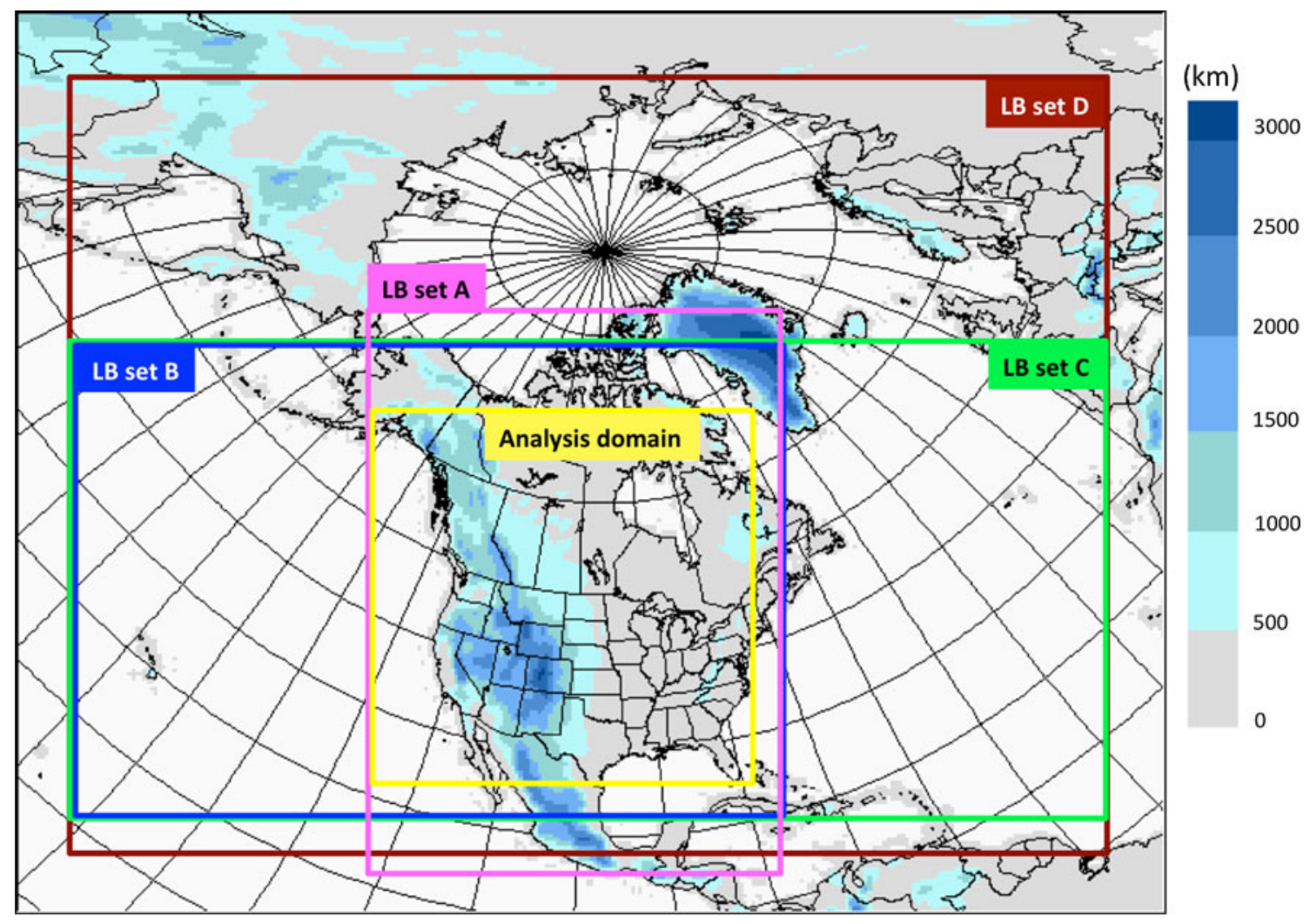

Fig. 1 LB domains of integration and topography. The picture shows only the inner domains, the nesting zones being excluded. The yellow square shows the common verification domain that is used in the comparative analysis 
Table 2 The LB simulations

\begin{tabular}{lllllll}
\hline Driving simulation & PBB (p0) & IBB1 (p1) & IBB2 (p2) & IBB3 (p3) & IBB4 (p4) & ERA-Interim (p5) \\
\hline LB set A & LBdAp0 & LBdAp1 & LBdAp2 & LBdAp3 & LBdAp4 & LBdAp5 \\
LB set B & LBdBp0 & LBdBp1 & LBdBp2 & LBdBp3 & LBdBp4 & LBdBp5 \\
LB set C & LBdCp0 & LBdCp1 & LBdCp2 & LBdCp3 & LBdCp4 & LBdCp5 \\
LB set D & LBdDp0 & LBdDp1 & LBdDp2 & LBdDp3 & LBdDp4 & LBdDp5 \\
\hline
\end{tabular}

and diffusion coefficients within a lateral sponge zones (Davies 1976; Robert and Yakimiw 1986; Yakimiw and Robert 1990). It is important to note that even if the sponge region is excluded from analysis, the nesting errors could affect the entire flow, due to transport across the RCM domain. Also, sometimes, the strong gradients from the downstream lateral boundaries are creating reflective nonmeteorological waves that are not sufficiently damped within the sponge zone and can affect the entire flow. Such nesting errors can evolve and interact in complex ways with the LBC errors. Large-scale spectral nudging (e.g., von Storch et al. 2000; Biner et al. 2000; Miguez-Macho et al. 2004; Castro et al. 2005) is an option designed to minimise this effect.

Second, the high resolution of an RCM permits a better representation of the regional forcings such as topography, coastlines and land-surface properties, as well as mesoscale dynamics, than in a coarse-resolution GCM. The question then is whether this a priori advantage of an RCM can lead to an improvement of the simulated climate over the domain and reduce the errors present in the driving LBC fields. It is important to remember however that the limited area of an RCM domain imposes an upper limit on the largest scales that are explicitly resolved by the model. Hence it is possible that, despite the a priori advantage of an RCM, a possible degradation of the large scales can ensue.

Using an extension of the perfect-model "Big-Brother (BB) Experiment" (Denis et al. 2002b) approach, Diaconescu et al. (2007) performed a detailed analysis of the Canadian RCM (CRCM) errors arising from LBC errors using an approach they called the "Imperfect Big-Brother (IBB) Experiment". The IBB approach permits to evaluate the CRCM errors resulting from nesting with LBC imperfections that mimic the typical errors of a GCM. Using a 2-D Discrete Cosine Transform (DCT) filter (Denis et al. 2002a), they separated large and small scales in order to evaluate the impact of errors in the driving fields on the large and small scales simulated by the CRCM. The analysis focused on the climate statistics of five February months over the East Coast of North America and the Western Atlantic. The study showed that the errors of the large-scale nesting fields (in mean sea level pressure and 850-hPa temperature) are reproduced by the CRCM large scales, without any important reduction or amplification. The LBC apparently exerted a high constraint on the
CRCM solution, imposing in CRCM the same large scales as in the driving fields. They also showed that the small scales developed in regions with errors in the large scales have errors as well, highlighting that the large scales precondition the small scales.

The study of Diaconescu et al. (2007) used RCM simulations performed over a relatively small domain of $100 \times 100$ grid points with a 45-km grid mesh and 10-point-wide nesting zone (for a free domain of $3,600 \times 3,600 \mathrm{~km}^{2}$ ). Increasing available computational resources now permit longer simulations over larger domains and longer periods. It is recognized that the domain size influences strongly the control exerted by the LBC. Studies with ensemble RCM simulations have shown that, as the domain size increases, the LBC exert less constraint on the RCM solution that may then diverge substantially from the driving fields (e.g. Giorgi and $\mathrm{Bi}$ 2000; Christensen et al. 2001; Rinke et al. 2004; Alexandru et al. 2007; Lucas-Picher et al. 2008a; Rapaić et al. 2011), a large domain of integration permitting the RCM to create its own weather sequence. A large domain hence favours the growth of inter-member (or internal) variability. In their study, Lucas-Picher et al. (2008b) showed that the magnitude of internal variability increases with the time that an air parcel spends inside the RCM domain, longer residence time (Lucas-Picher et al. 2008b) allowing more time for the development of differences in flow evolution (Nikiéma and Laprise 2011; Diaconescu et al. 2012).

How LBC errors influence very large domain RCM simulations? On the one hand, nesting errors will be larger with large domains and risk degrading the RCM-simulated large scales. On the other hand, the RCM's high resolution permitting a better representation of the climate, the use of larger domains could lead to an improvement of the large scales provided as LBC, in spite of the handicap caused by the nesting errors. The studies of Mesinger et al. (2002, 2012) and Veljovic et al. (2010) indicate that such an improvement may be possible when an RCM is run over a sufficiently large domain that allows escaping somewhat from the negative influence of the LBC errors. They presented simulations with the Eta RCM driven over a period of 32 days by members of an ensemble of forecasts by the European Centre for Medium-range Weather Forecasts (ECMWF), evaluated against ECMWF analyses. The Eta RCM was run over a large domain of 12,000 $\times 7,111 \mathrm{~km}^{2}$. 
Fig. 2 Climatic mean of the geopotential field at $500 \mathrm{hPa}$ simulated by PBB, ERA-Interim and the four IBBs. The yellow square shows the domain that is used in the comparative analysis with the LB simulations
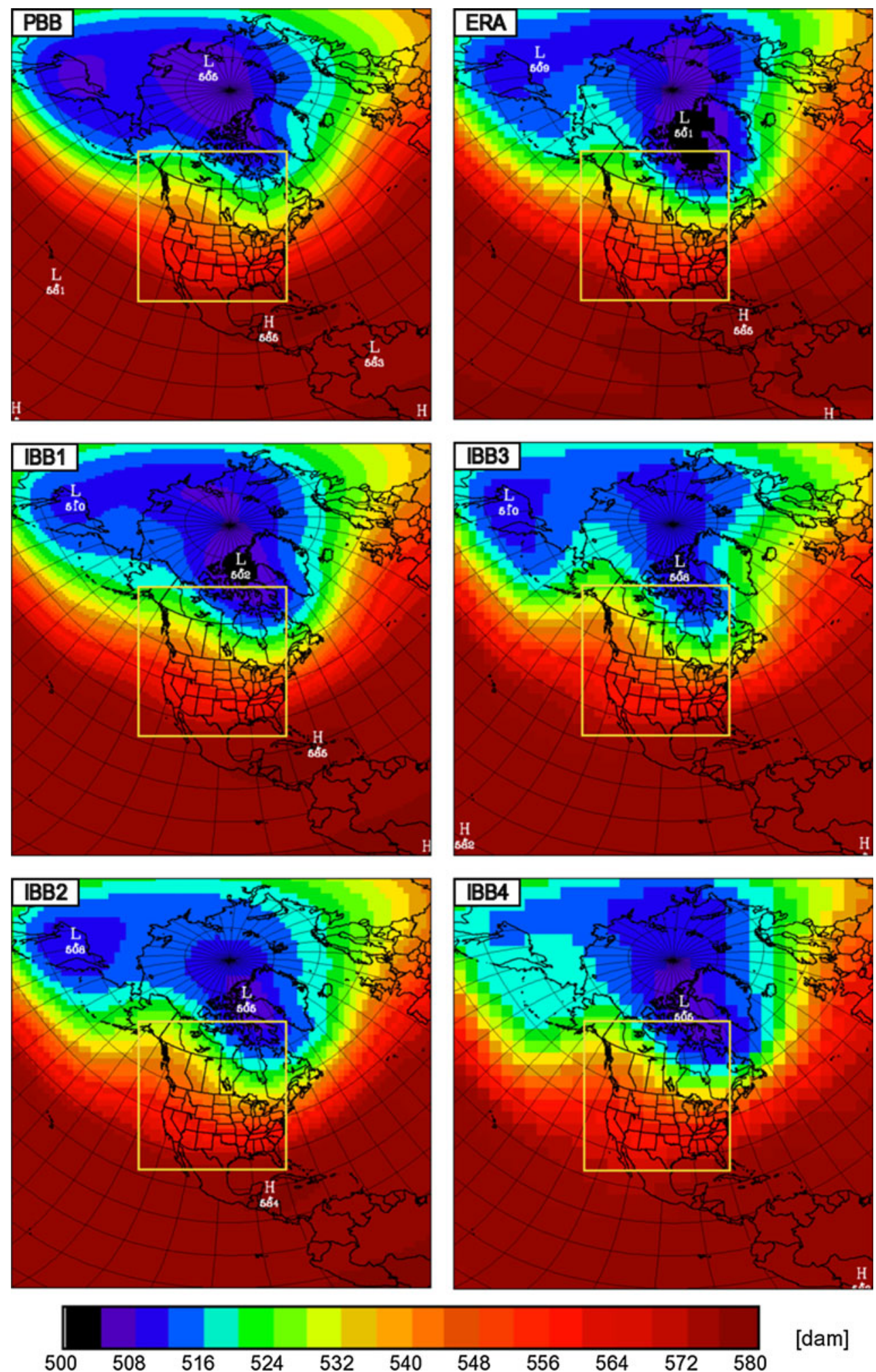

[dam]
Their study showed that the Eta RCM forecast presents periods with improvement, compared to the driving model, in the position of the strongest winds at the jet-stream level. They argued that the improvement in the large-scale solution is due to an improved definition of Rocky
Mountains topography in the high-resolution RCM compared to that in the ECMWF driving model and to the use of Eta coordinate in the RCM. Because two different formulations were used for the nested and driving models, it is difficult to attribute the improvement either to a better 
Fig. 3 Average intra-seasonal standard deviation of the geopotential field at $500 \mathrm{hPa}$ simulated by PBB, ERA-Interim and the four IBBs. The yellow square shows the domain that is used in the comparative analysis with the LB simulations
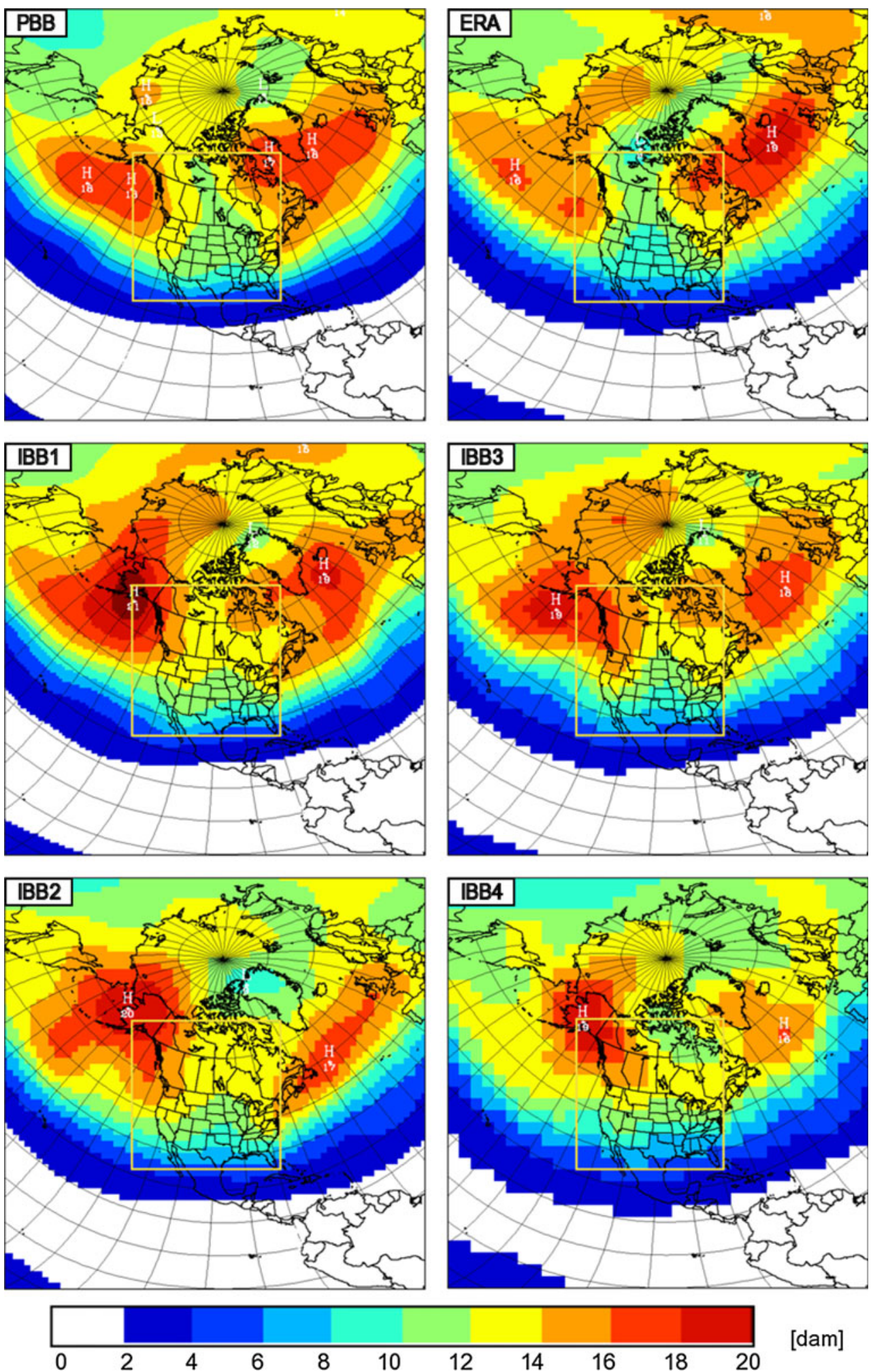

[dam]

representation of the topography or to other differences between the two models. There are also predictability issues for deterministic forecasts at such long time range. Nonetheless these studies provide motivation to investigate whether such improvement of large-scale patterns could be obtained in RCM climate statistics as well as in forecasts.

The LBC errors impact on RCM simulations constitutes a complex issue that calls for further investigation. The 
Fig. 4 The PBB field and the differences between the four $\mathrm{LB}$ fields driven by $\mathrm{PBB}$ (LBdAp0, LBdBp0, LBdCp0, LBdDp0) and the PBB field, computed for the large-scale stationary component of precipitation rate (mm/day)
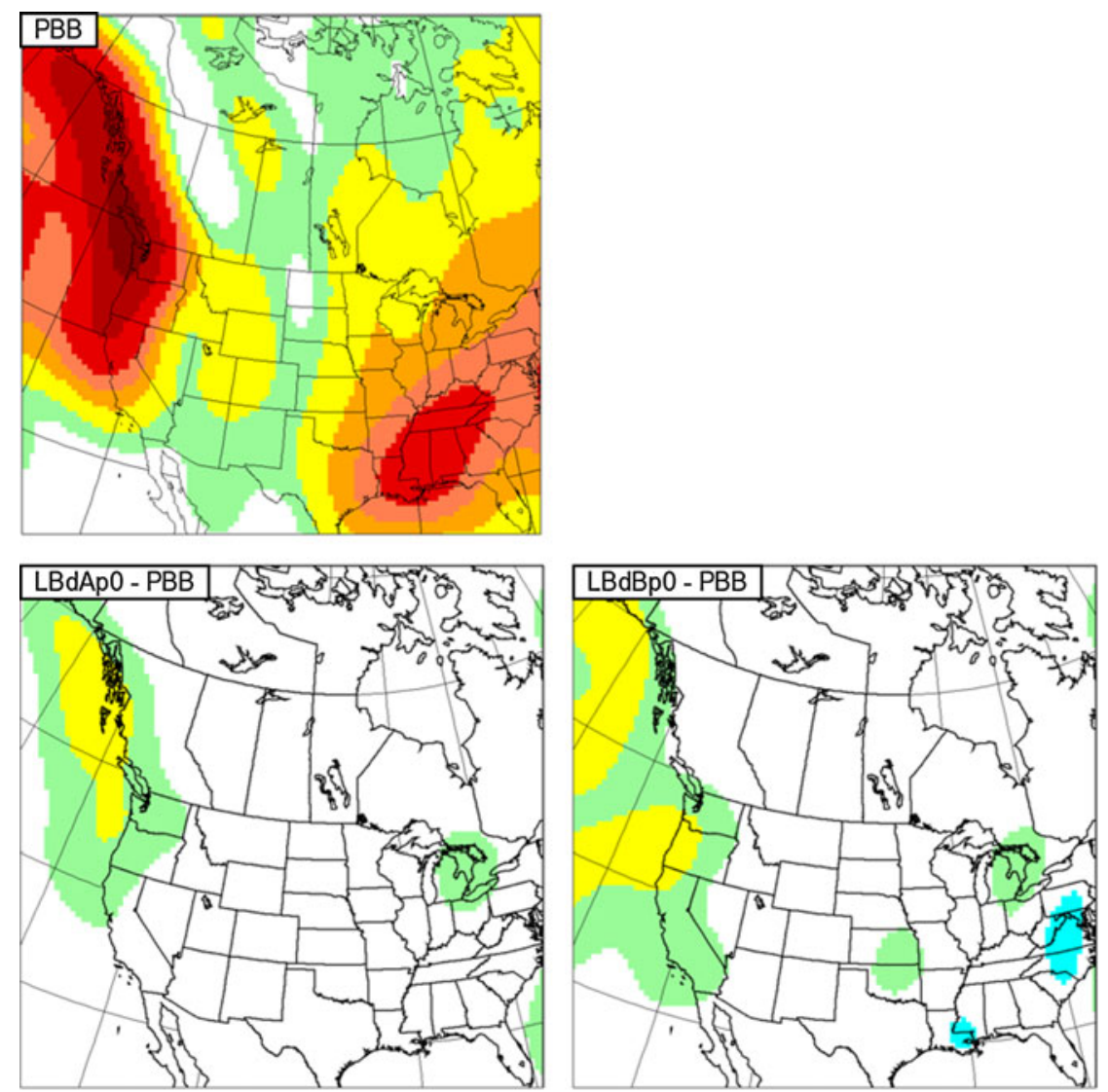

8.0
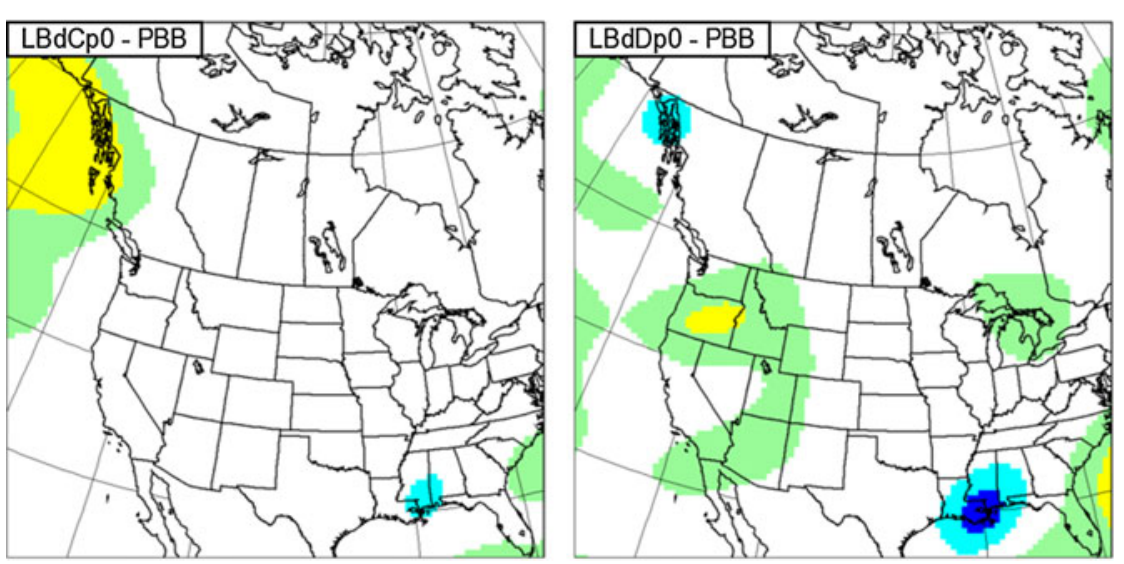

1.0

0.5

$-0.5$

$-1.0$

$-2.0$

$-3.0$

$-4.0$
Table 3 Spatial correlation coefficients of the large-scale precipitation of LBs driven with PBB fields

\begin{tabular}{lllll}
\hline & LBdAp0 & LBdBp0 & LBdCp0 & LBdDp0 \\
\hline Time mean & 0.997 & 0.988 & 0.989 & 0.986 \\
$\begin{array}{l}\text { Intra-seasonal transient } \\
\text { component }\end{array}$ & 0.998 & 0.991 & 0.987 & 0.979 \\
\hline
\end{tabular}

main objective of this paper is to investigate the sensitivity of RCM-simulated large scales to domain size and LBC errors. The fifth-generation Canadian RCM (CRCM5) will be run over successively larger domains centred on North America. In order for the results not to be influenced by errors specific to this model, the IBB framework is followed, using two configurations of the exact same model, one as driving global model and the other as nested limited-area model, the only differences being the domain of integration and the resolution. The study focuses on the wintertime statistics over the North American continent. The design of the experiment and a brief description of the model are presented in Sect. 2 . Section 3 gives a general description of the driving fields. Sections 4 and 5 present the comparative analysis of RCM large- and small-scale errors, respectively. The conclusions are presented in Sect. 6. 
Fig. 5 The differences between the four LB fields driven by ERA-Interim (LBdAp5, LBdBp5, LBdCp5, LBdDp5) and the PBB field, computed for the large-scale stationary component of precipitation rate $(\mathrm{mm} /$ day)
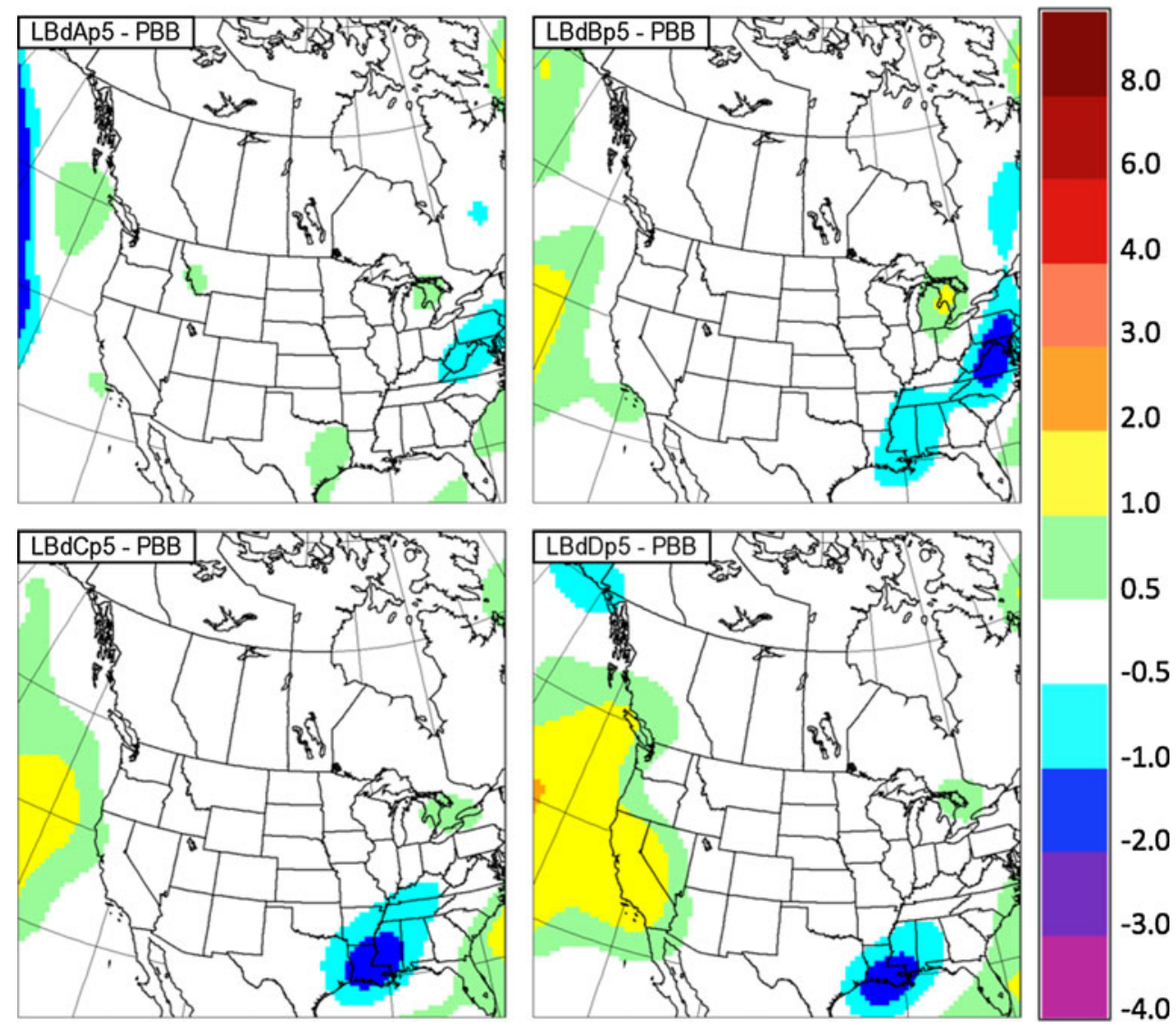

\section{Model description and experimental set-up}

The $\mathrm{BB}$ experiment is a perfect-model approach designed to isolate the errors that are specific to the nesting process from other model errors. The full version of this approach (e.g. Laprise et al. 2008) consists in performing a high-resolution global model simulation, named the Big-Brother (BB) simulation, which will serve two purposes. It will provide so-called "perfect" LBC for driving limited-area simulations, named the Little-Brother (LB) simulations, which will be run at the same high resolution as the global simulation. The BB simulation will also serve as virtual-reality reference against which the limited-area simulations will be evaluated. Given that the BB and LB simulations use the same resolution, physical parameterisations, dynamics and numerical discretisations, the differences in their climates will clearly be due to nesting errors as defined previously. Optionally, the LBC supplied to the LB by the BB simulations could be filtered by removing their small scales in order to assess the skill of the LB at reconstructing the small scales when driven by large-scale only LBC.

Due to the high computational resources required for performing high-resolution global simulations for long periods, the vast majority of previous BB studies (e.g. Denis et al. 2002b, 2003; Antic et al. 2004; Dimitrijevic and Laprise 2005; Herceg et al. 2006; Diaconescu et al.
2007; Køltzow et al. 2008; Leduc and Laprise 2009; Leduc et al. 2011; Rapaić et al. 2011) have used a "poor-man" version of $\mathrm{BB}$ experiment, generating the BB simulation by running an RCM over a large domain instead of a global model. An exception is the study of Radu et al. (2008) who used the full version of the BB experiment with ALADIN regional and ARPEGE global models.

As described, the high-resolution $\mathrm{BB}$ simulation provides a set of "perfect" $\mathrm{LBC}$ for the LB simulations, and hence it could also be called a "Perfect Big Brother" (PBB). In order to analyze the effect of the errors in the LBC driving an RCM, Diaconescu et al. (2007) extended the "poor man" BB experiment by performing, in addition to the PBB, several "Imperfect Big Brother" (IBB) simulations that intend to mimic the typical GCM errors. IBB simulations were run at a lower horizontal resolution then the PBB and hence provided a set of "imperfect" LBC for the LB simulations, while the PBB was used as reference for evaluating the errors of the driving IBB and the errors of the ensuing LB simulations.

In this study, we used the full version of the IBB experiment. The PBB, IBB and LB simulations were obtained by running versions of Canadian Global Environmental Multiscale (GEM) model developed at Meteorological Service of Canada for Numerical Weather Prediction (NWP) (Côté et al. 1998; Yeh et al. 2002). 
Fig. 6 The IBB1 field and the differences of IBB1 and the four LB fields driven by IBB 1 (LBdAp1, LBdBp1, LBdCp1, LBdDp1) from the PBB field, computed for the large-scale stationary component of precipitation rate $(\mathrm{mm} /$ day)
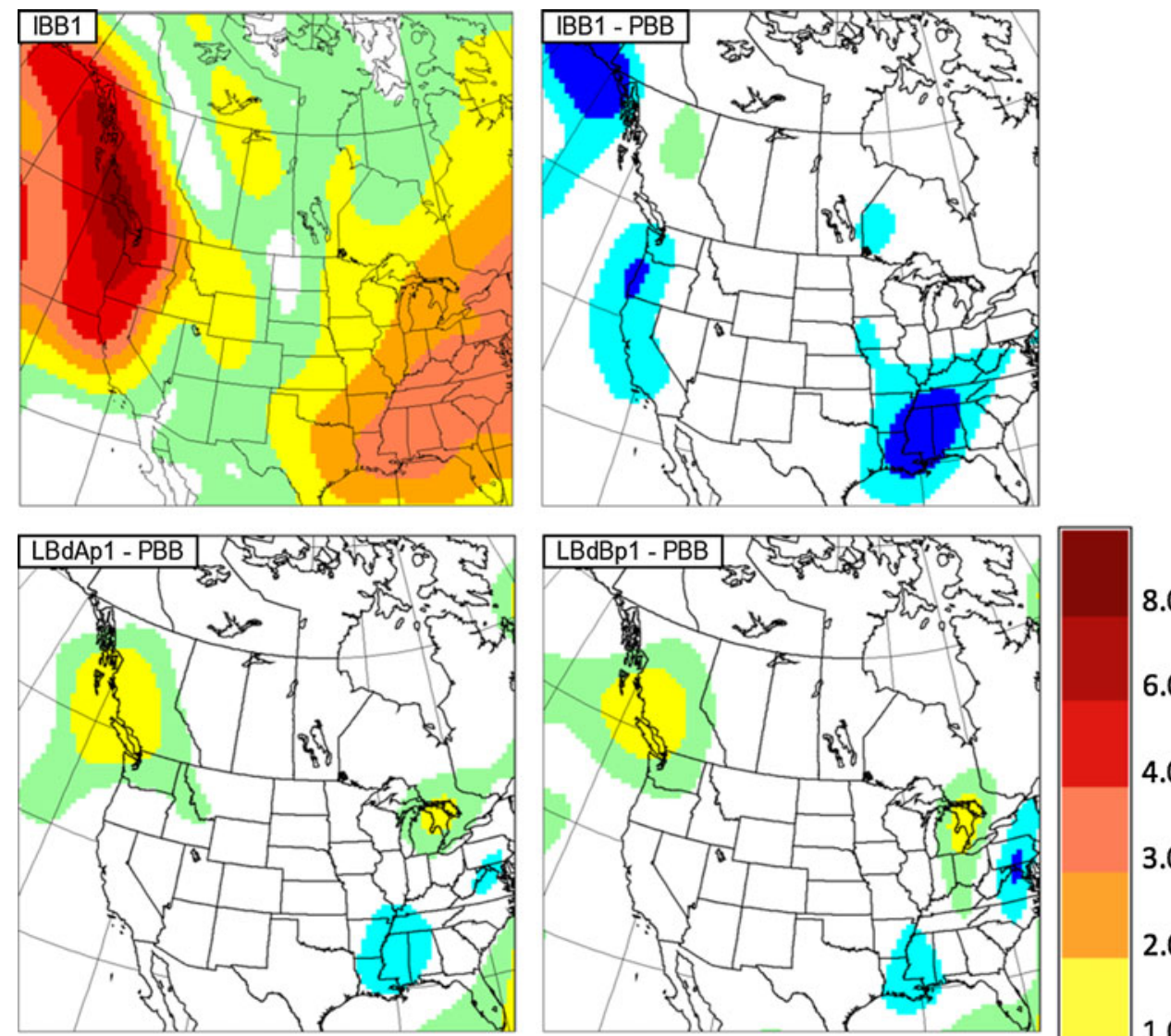

8.0
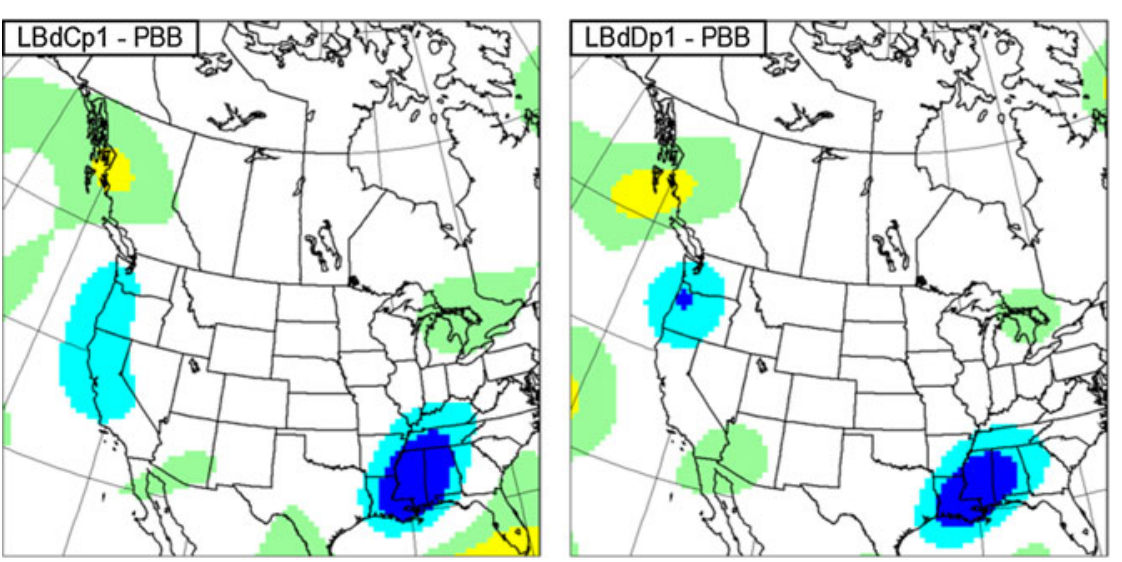

1.0

0.5

$-1.0$

$-2.0$

$-3.0$
The GEM model supports three configurations: uniform latitude-longitude global, stretched-grid global and nested limited-area. The PBB and IBB simulations were performed with the uniform global climate version, GEMCLIM v.3.3.2.1. The LB simulations were obtained with a limited-area version of GEM that constituted an interim version of the developmental fifth-generation Canadian Regional Climate Model (CRCM5; Zadra et al. 2008). The CRCM5 is developed by the ESCER Centre, in collaboration with the Meteorological Service of Canada, as a climatic version of the limited-area model used in NWP.

The global (GEMCLIM) and regional (CRCM5) models share the same dynamical and physical cores. In both configurations, the model can use either the fully elastic non-hydrostatic or hydrostatic equations, solved with a two-time-level implicit semi-Lagrangian marching scheme. The spatial discretization operates on a staggered Arakawa C-grid (Arakawa and Lamb 1977). The model uses a terrain-following vertical coordinate based on normalized hydrostatic pressure (Laprise 1992). In our configuration, all simulations used the same rotated horizontal latitudelongitude grid with the equator shifted over the middle of the North American continent, and used 64 levels in the vertical with an uppermost level at $2 \mathrm{hPa}$. Physical parameterisations include the Canadian Land-Surface Scheme (CLASS v3.5; Verseghy et al. 1993; Verseghy 
Fig. 7 The IBB2 field and the differences of IBB2 and the four LB fields driven by IBB2 (LBdAp2, LBdBp2, LBdCp2, LBdDp2) from the PBB field, computed for the large-scale stationary component of precipitation rate $(\mathrm{mm} /$ day)
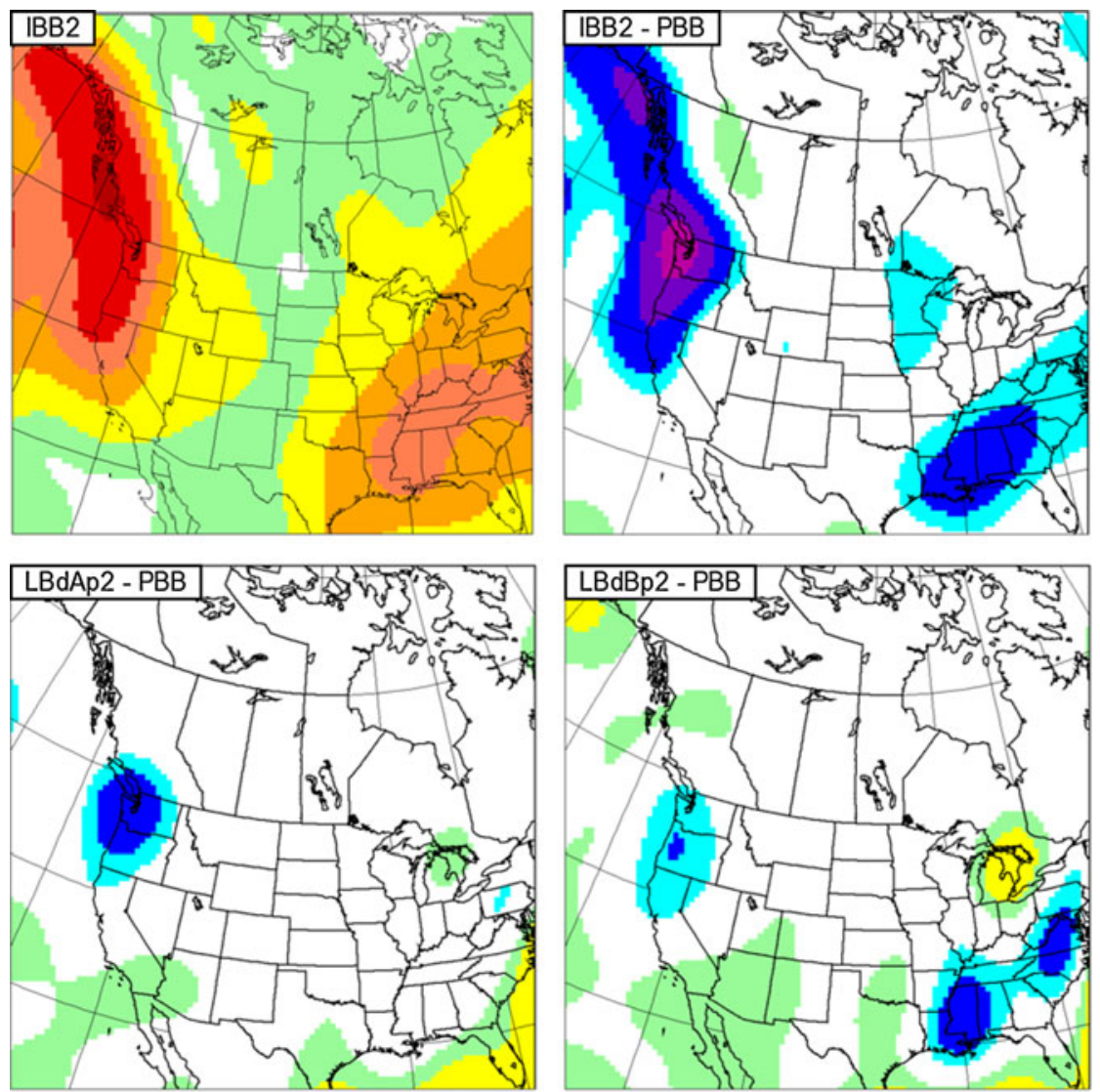

8.0

6.0

4.0

3.0

2.0
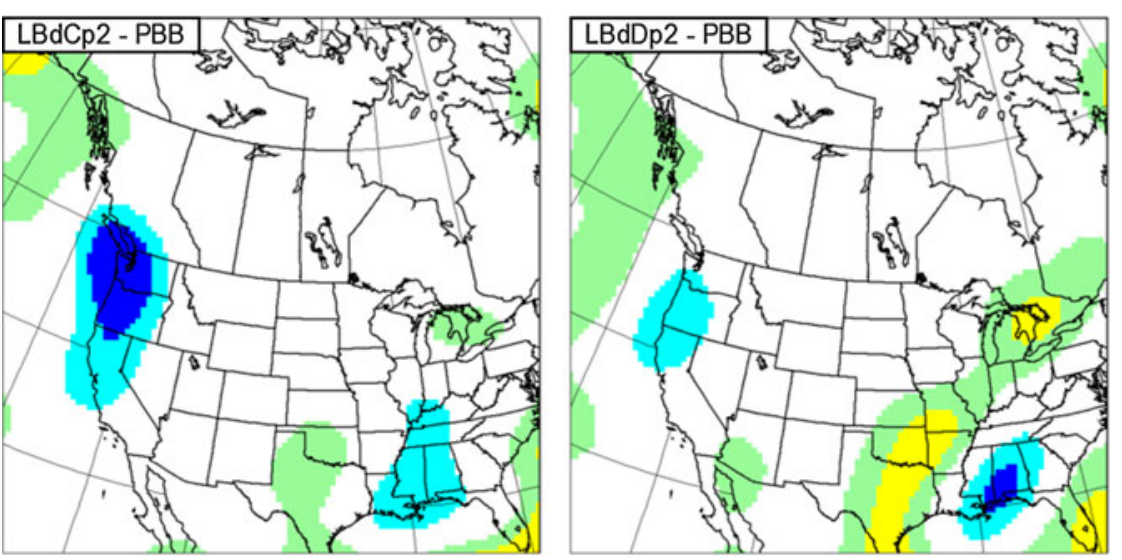

1.0

0.5

$-0.5$

$-1.0$

$-2.0$

$-3.0$

$-4.0$
2000), the deep-convection scheme of Kain and Fritsch (1990), the Kuo transient shallow-convection scheme (Kuo 1965; Bélair et al. 2005), the large-scale condensation scheme by Sundqvist et al. (1989). The radiation package for solar and terrestrial radiation is based on the correlated$\mathrm{K}$ approach ( $\mathrm{Li}$ and Barker 2005) and the Liang and Wang (1995) monthly ozone climatology was also used. Subgridscale orographic gravity-wave drag is due to McFarlane (1987) and low-level orographic blocking is described in Zadra et al. (2003). For all simulations, sea-surface temperature and sea-ice surface boundary conditions were interpolated from the Atmospheric Model Intercomparison Project v2 (AMIP2; Gleckler 1996) available on a $1^{\circ}$ latitude-longitude grid for monthly mean values. The model outputs were archived at 3-hourly intervals.

Five BB simulations were done with GEMCLIM. The first used a high resolution of $0.45^{\circ}$ and a timestep of $20 \mathrm{~min}$; this simulation is referred to as the Perfect BigBrother (PBB) and it will serve as reference. Four Imperfect Big-Brother (IBB) simulations were also obtained with GEMCLIM using coarser horizontal resolutions that are typical for the GCMs used in climate-change projection studies: $0.9^{\circ}$ (IBB1), $1.8^{\circ}$ (IBB2), $2.25^{\circ}$ (IBB3) and $3.6^{\circ}$ (IBB4) (see Table 1). The timestep, the horizontal diffusion and the radiation calculation interval were adjusted corresponding to the horizontal resolution. The PBB- and 
Fig. 8 The IBB3 field and the differences of IBB3 and the four LB fields driven by IBB3 (LBdAp3, LBdBp3, LBdCp3, LBdDp3) from the PBB field, computed for the large-scale stationary component of precipitation rate $(\mathrm{mm} /$ day $)$
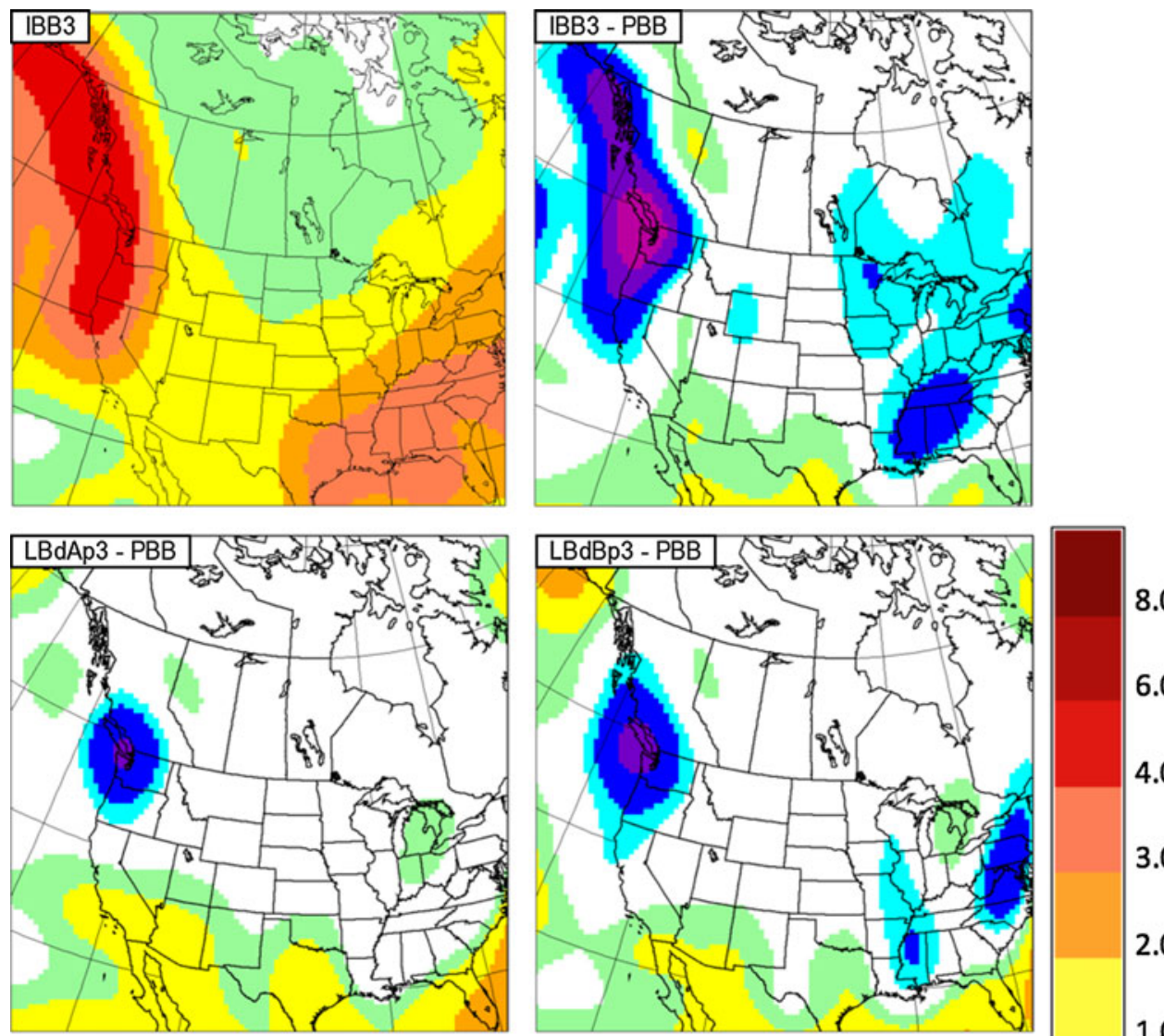

8.0 6.0 4.0
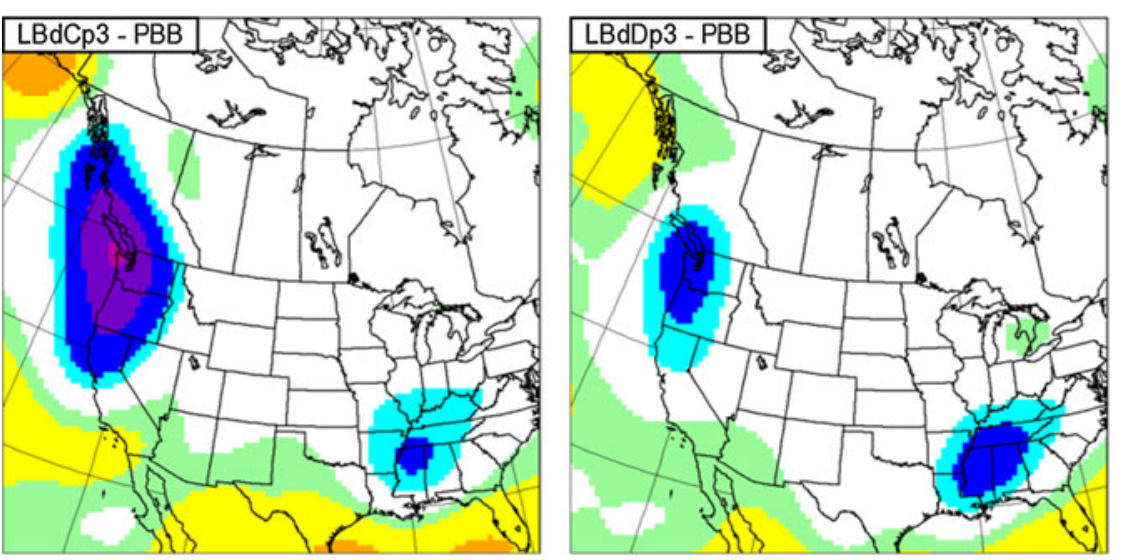

1.0

0.5 $-0.5$

$-1.0$

$-2.0$

$-3.0$
IBB-simulated data were used for driving the LB CRCM5 simulations. The only difference between the LB and PBB simulations is the nested regional versus global configurations of the $\mathrm{LB}$ and $\mathrm{PBB}$, respectively.

CRCM5 is a one-way nested model. It uses a 10 grid-point nesting zone, inspired by the work of Davies (1976) and adapted by Robert and Yakimiw (1986) and Yakimiw and Robert (1990). The LBC provided by the PBB and the four IBBs consist in the fields of sea-level pressure, temperature, horizontal wind, specific humidity, and liquid and solid water in the atmosphere. The BB fields were archived at every $3 \mathrm{~h}$ on model levels, and for driving the CRCM5, they are linearly interpolated in time to every CRCM5 time step, and spatially interpolated from IBB to LB grid and levels. A supplementary set of LBC is provided by the ERA-Interim ECMWF reanalysis; these are provided at every $6 \mathrm{~h}$ on 22 pressure levels and they are interpolated as required to provide $\mathrm{LBC}$ for CRCM5. In the virtual-reality world in which the $\mathrm{PBB}$ represents the reference, ERA-interim constitutes a kind of fifth IBB whose apparent imperfections in fact reflect the inadequacy of the $0.45^{\circ}$ version of GEMCLIM to reproduce the climate statistics of ERA-Interim.

For each set of LBC (the PBB, the four IBBs and ERAInterim), four LB simulations were made over increasingly 
Fig. 9 The IBB4 field and the differences of IBB4 and the four LB fields driven by IBB4 (LBdAp4, LBdBp4, LBdCp4, LBdDp4) from the PBB field, computed for the large-scale stationary component of precipitation rate $(\mathrm{mm} / \mathrm{day})$
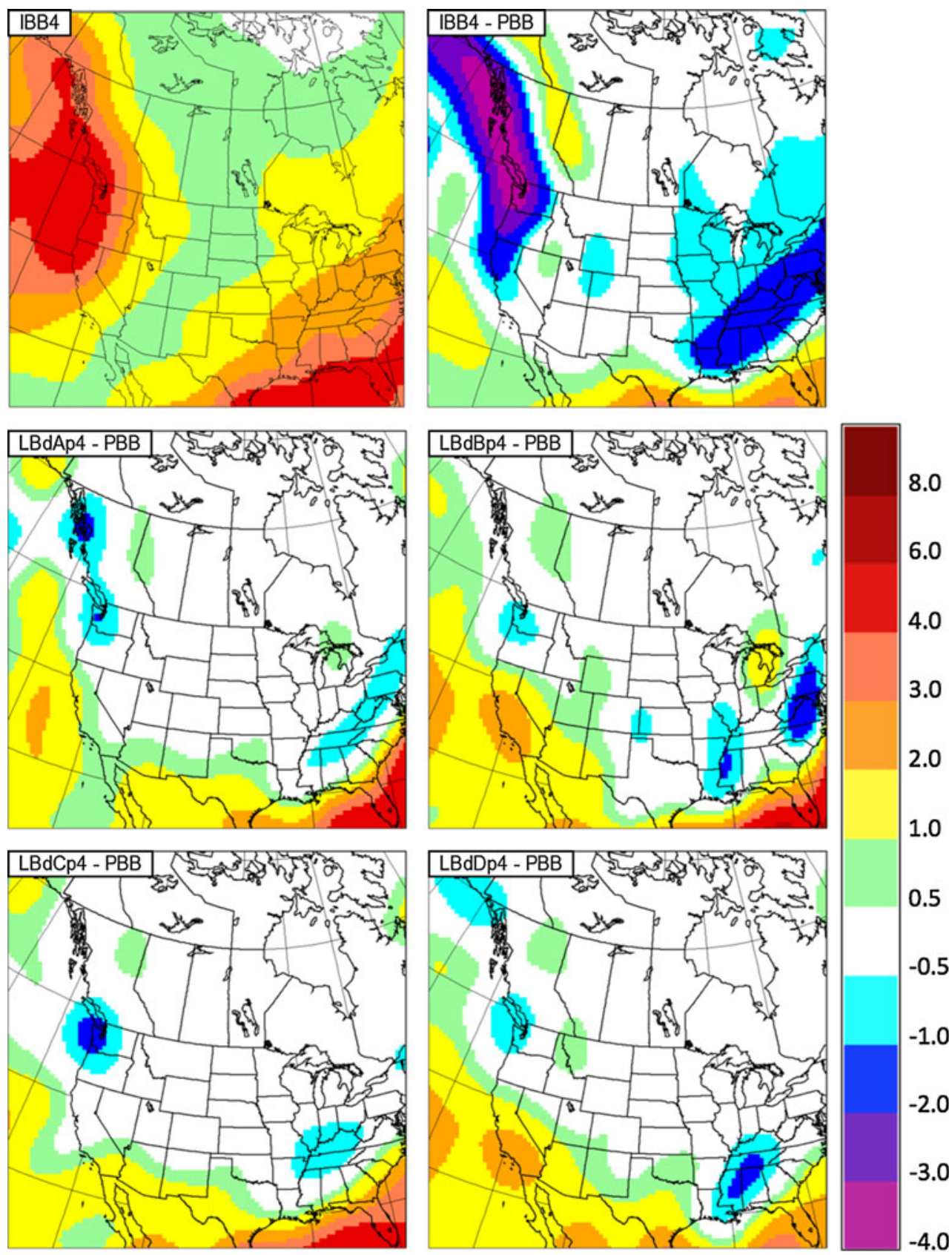

0.5

larger domains enclosing the North American Continent; the domains of integration, noted with $\mathrm{A}, \mathrm{B}, \mathrm{C}$ and $\mathrm{D}$, are illustrated in Fig. 1. Note that the 10-grid-point nesting zone is excluded from the figure that displays only the inner, free domain. Domain A (in magenta) has an inner domain of $108 \times 146$ grid points covering the North American Continent; this is approximately 3 times larger then the inner domain used in Diaconescu et al. (2007). The domain B (in blue) has an inner domain of $188 \times 126$ grid points and covers the North American continent plus a large part of the North East Pacific Ocean. The domain C (in green) extends the domain $\mathrm{B}$ by including also a large part of the North West Atlantic Ocean and has an inner domain of $272 \times 126$ grid points. Finally domain D (in red) extends domain $\mathrm{C}$ by including the Arctic Ocean; it has an inner domain of $272 \times 206$ grid points, which is 3.5 times larger then the domain $\mathrm{A}$. The figure also shows, in yellow, the common verification domain that will be used in the analysis. LB simulations are named (see Table 2) following a convention that gives the domain of integration (A, B, C or D) and a number that is associated with the set of LBC (0 stands for PBB, 1 for IBB1, 2 for IBB2, 3 for IBB3, 4 for IBB4 and 5 for ERA-Interim). The name is in the form $\operatorname{LBd}\{$ domain $\}$ p driving-model number $\}$; for example, $\mathrm{LBdCp} 3$ represents the $\mathrm{LB}$ simulation run over domain $\mathrm{C}$ using as driving LBC the IBB3 fields. This 
Fig. 10 Large-scale interseasonal transient component of precipitation rate $(\mathrm{mm} /$ day $)$ as simulated by PBB, IBB4 and the four LBs driven by IBB4
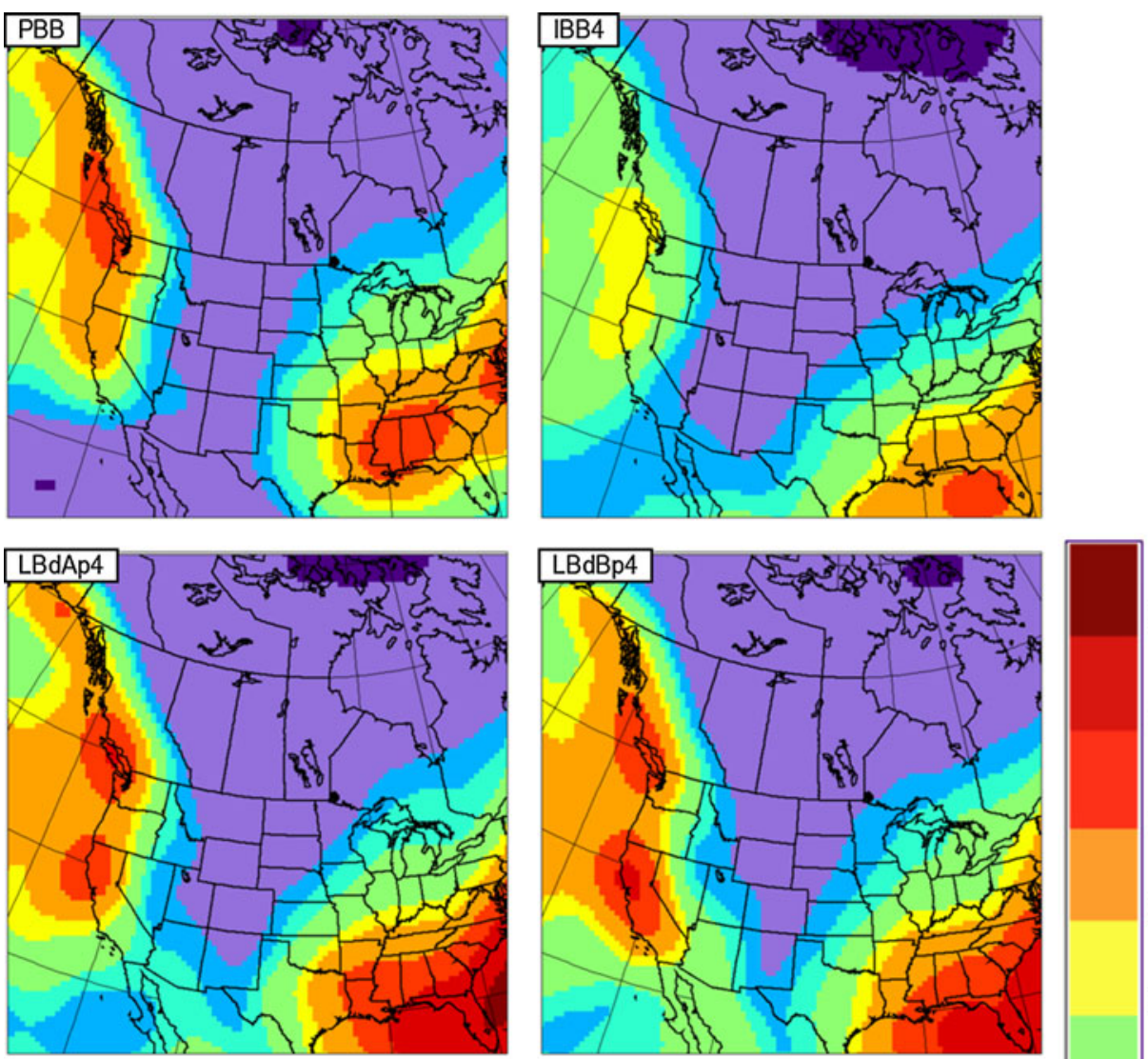

15.0

12.0

10.0

8.0

7.0
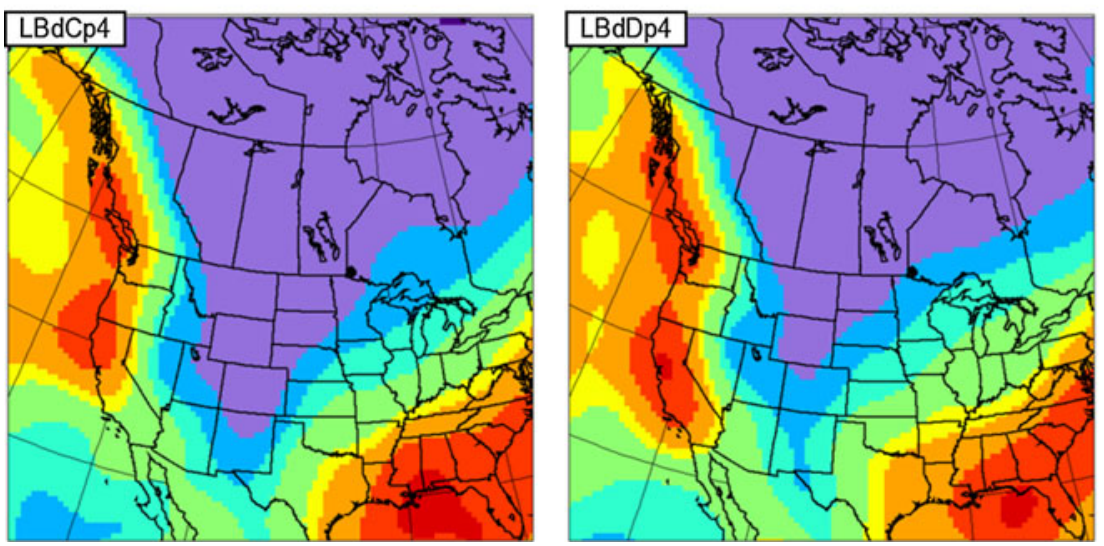

5.0

4.0

3.0

1.0

0.5

0.0 convention will be used in the most part of the figures presented in the paper.

All simulations (PBB, 4 IBBs and $6 \times 4 \mathrm{LB}$ ) used for initialization the same land surface fields that were provided by a prior monthly climatology generated with GEMCLIM at $1^{\circ}$ horizontal resolution. They used the same atmospheric initial conditions from the ERA-Interim ECMWF reanalysis. The period of integration corresponds to 4 months, starting on 1st November at 00:00 UTC and ending the 1st March at 00:00 UTC, for five consecutive years 1996/1997, 1997/1998, 1998/1999, 1999/2000 and
2000/2001. The months of November are considered as spin-up and are not included in statistics; only the threemonth winter periods (December-January-February) are analysed.

\section{The driving-model and ERA-Interim fields}

As mentioned previously, in this study, $\mathrm{PBB}$ is regarded as the "perfect" simulation representing the virtual reference to which the other simulations will be compared. The 

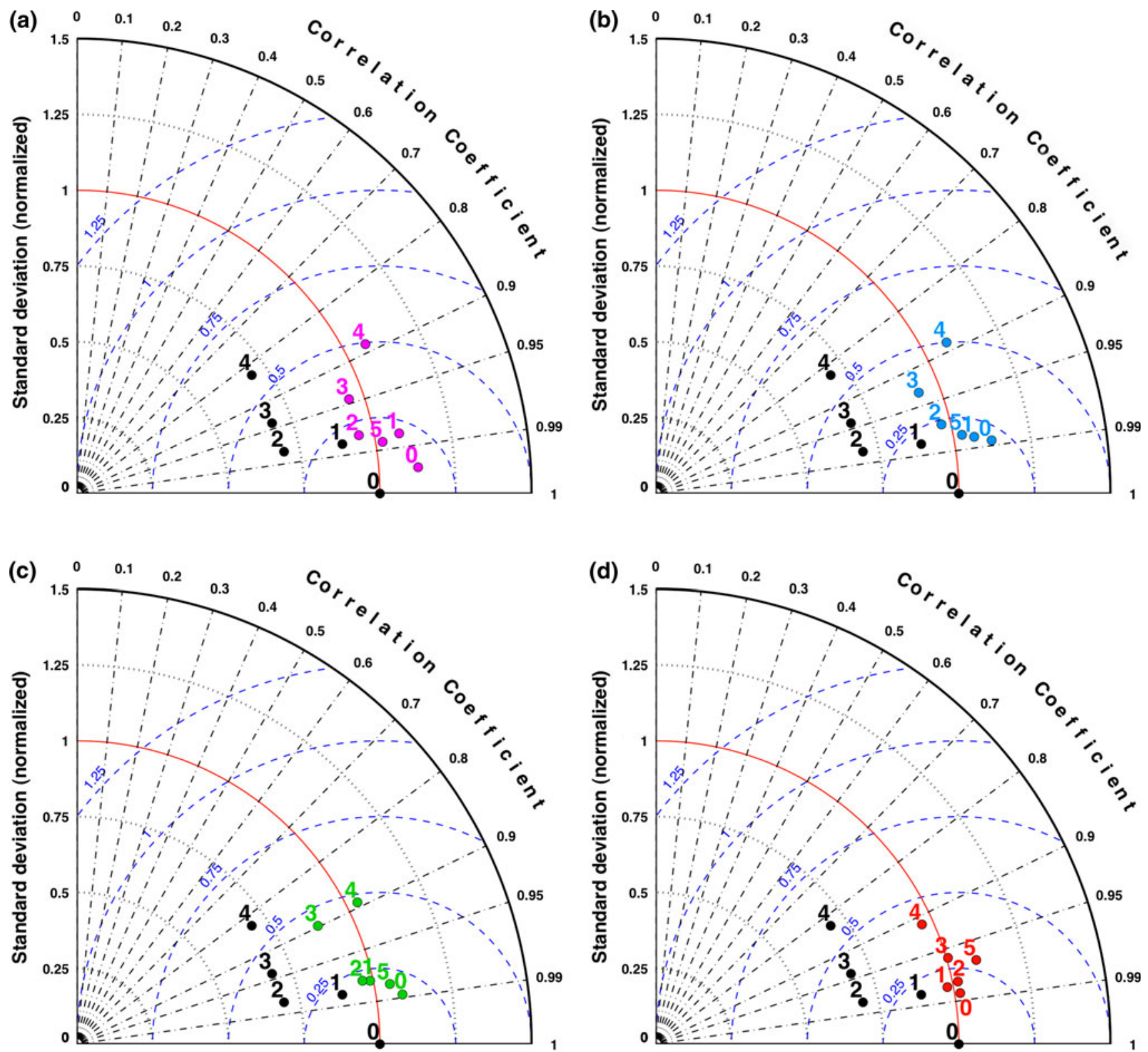

\section{LBs domain A
LBs domain B domain C
LBs domain D}

Fig. 11 Taylor diagrams showing the large-scale precipitation-rate errors in the stationary component of IBBs, ERA and LBs

statistics focus on the 5-year time mean and intra-seasonal standard deviation, computed as follow.

For any meteorological field $\left(F_{y}\right)$, and for each year $(y)$, the seasonal mean (noted $\overline{F_{y}}$ ) is computed as a time average over the period December, January and February, and the intra-seasonal standard deviation as

$\sigma_{y}=\sqrt{\left(F_{y}-{\overline{F_{y}}}^{s}\right)^{2}}$

The 5-year mean is computed as the average of the seasonal means
$\bar{F}^{y}=\frac{1}{5} \sum_{y=1}^{5}{\overline{F_{y}}}^{s}$,

and the average intra-seasonal standard deviation as

$\sqrt{{\overline{\sigma_{y}^{2}}}^{y}}=\sqrt{\frac{1}{5} \sum_{y=1}^{5} \sigma_{y}^{2}}$.

Figure 2 shows the mean 500-hPa geopotential for PBB, ERA-Interim and the four IBBs. The GEMCLIMsimulated fields are presented on their original grid, 

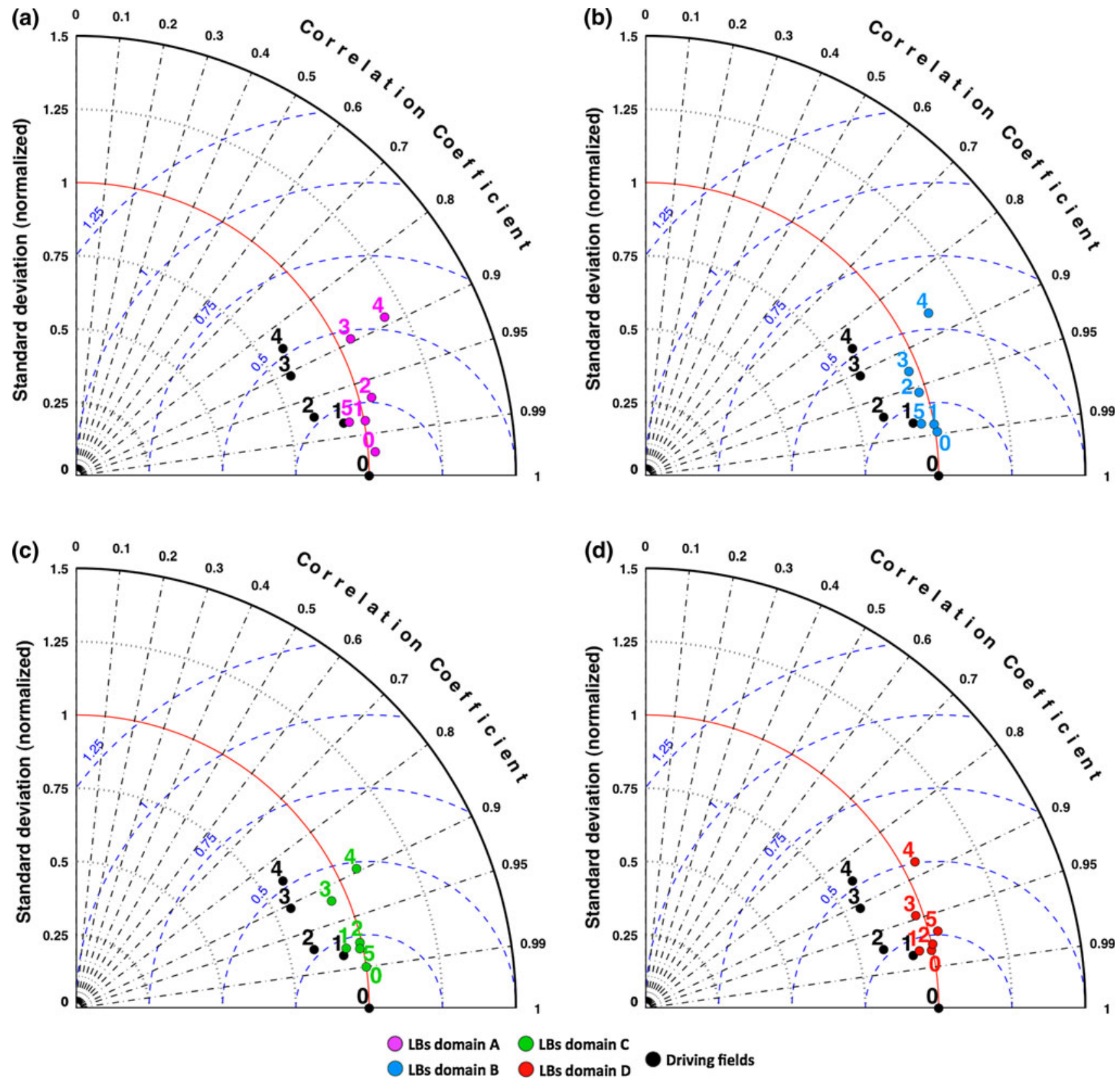

Fig. 12 Taylor diagrams showing the large-scale precipitation-rate errors in the intra-seasonal transient component of IBBs, ERA and LBs

while the ERA-Interim fields (originally at $2^{\circ} \times 2^{\circ}$ on a latitude-longitude non-rotated grid) are interpolated on the GEMCLIM rotated grid at $2^{\circ} \times 2^{\circ}$. The climatological features of troughs over Hudson Bay and Eastern Siberia and a ridge over Western North America are clearly seen, with different intensities. The Eastern Siberia trough in PBB is similar to that in ERA-Interim (506 and 509 dam, respectively), while the trough is shallower in the coarser resolution IBBs, reaching 515 dam in IBB4, which is probably due to the smoother topography in the coarser resolution IBB simulations. The depth of the Hudson Bay trough on the other hand is fairly robust, with small variations from 501 dam in ERA-Interim to 508 in IBB3; its position however occurs at more northerly latitudes in PBB compared to ERA-Interim and the four IBBs. Over Western North America, the ridge extends further to the North in IBB2, IBB3 and IBB4.

The average intra-seasonal standard deviation of the $500-\mathrm{hPa}$ geopotential is presented in Fig. 3. The two important maxima are associated with the primarily storm tracks, one over the Eastern North Pacific Ocean and one over the North Atlantic Ocean. The PBB resembles most 
Fig. 13 LB errors versus BB errors for large-scale precipitation rate in $(\mathbf{a}, \mathbf{c})$ the stationary component and $(\mathbf{b}, \mathbf{d})$ the intra-seasonal transient component. Panels a, b shows the RMS errors, while panels c, $\mathbf{d}$ presents the spatial standard deviation normalized by PBB spatial standard deviation. The asterisks stand for LBs nested by $\mathrm{PBB}$, the triangles for LBs nested by IBB1, the squares for LBs nested by IBB2, the stars for LBs nested by IBB3 and the circles for LBs nested by IBB4
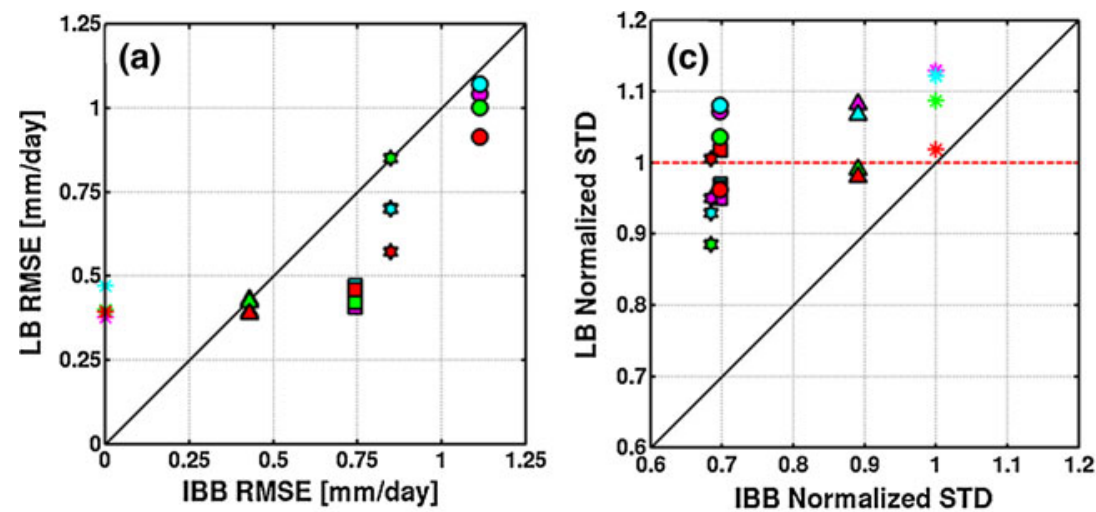

* LBdAp0

$\triangle$ LBdAp1

LBdAp2

LBdAp3

LBdAp4

LBdBp0

LBdBp1

LBdBp2

LBdBp3

LBdBp4

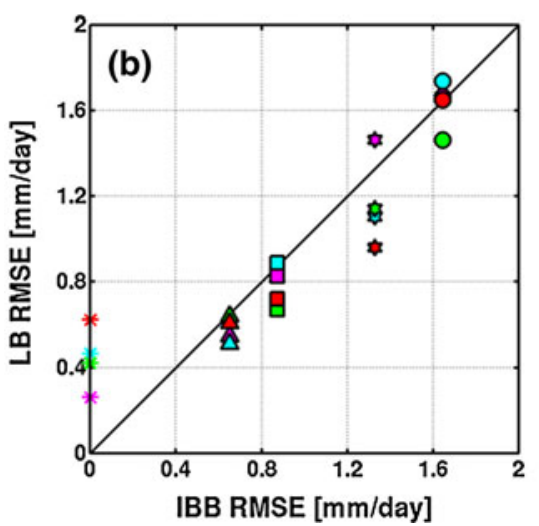

LBdCp0

$\triangle$ LBdCp1

LBdCp2

LBdCp3

LBdCp4

* LBdDp0

$\triangle$ LBdDp1

LBdDp2

LBdDp3

LBdDp4 closely to ERA-Interim, while the four IBBs present several important differences. The Pacific maximum is extending over the Alaska and Rocky Mountains in the IBB simulations, with larger amplitude than in PBB and ERA-Interim. The minimum over central North America is less well defined in the IBB simulations. The PBB North Atlantic maximum is close to the ERA-Interim, although the New England maximum is somewhat too intense. This New England maximum is also too intense in IBB1, while it is absent in the coarser resolution IBB. Overall, Figs. 2 and 3 show that PBB represents well the atmospheric general circulation, while the low-resolution IBBs present different degrees of errors typical of GCMs.

A fair comparison between the different experiments (PBB, ERA-Interim, IBBs and LBs) demands that all results be on the same grid. The fields were first interpolated onto the $0.45^{\circ}$-mesh grid of the smallest LB domain, A. Then, a scale decomposition using the DCT filter (Denis et al. 2002a) is applied in order to extract the same large scales for all simulations. Considering that in these experiments IBB4 has the coarsest mesh $\left(3.6^{\circ} \times 3.6^{\circ}\right)$, which corresponds to shortest resolved scales of about $\lambda \cong 2 \Delta x \cong 800 \mathrm{~km}$, we used a band-pass filter with a gradual cut-off that excludes all wavelengths shorter than $957 \mathrm{~km}$ and preserves all those longer than $1,116 \mathrm{~km}$, and have a gradual squared cosine transition in between. The small scales are computed as the difference between the non-filtered field and the large-scale field. All the fields were finally cut to the analysis domain (the yellow square in Fig. 1), and the statistics were computed separately for the large- and the small-scale components. In this paper, we focus mainly on the large-scale statistics, the stationary component being represented by the 5-year seasonal mean (Eq. 2), while the intra-seasonal transient component is represented by the 5-year average intra-seasonal standard deviation (Eq. 3).

\section{The impact of the LBC errors on the LB-simulated large scales}

Three fields are chosen for the comparison: precipitation, specific humidity and zonal wind component. The last two fields are involved directly in the nesting process and the LB errors in these fields are the direct reflection of the LBC errors. On the other hand, precipitation is not directly involved in the nesting process; hence LB precipitation errors will be an indirect effect of LBC and simulation errors.

\subsection{Large-scale precipitation}

Figure 4 presents the large scale of the mean winter precipitation field, for the reference PBB (the first panel) and 
(a) Large-scale stationary component

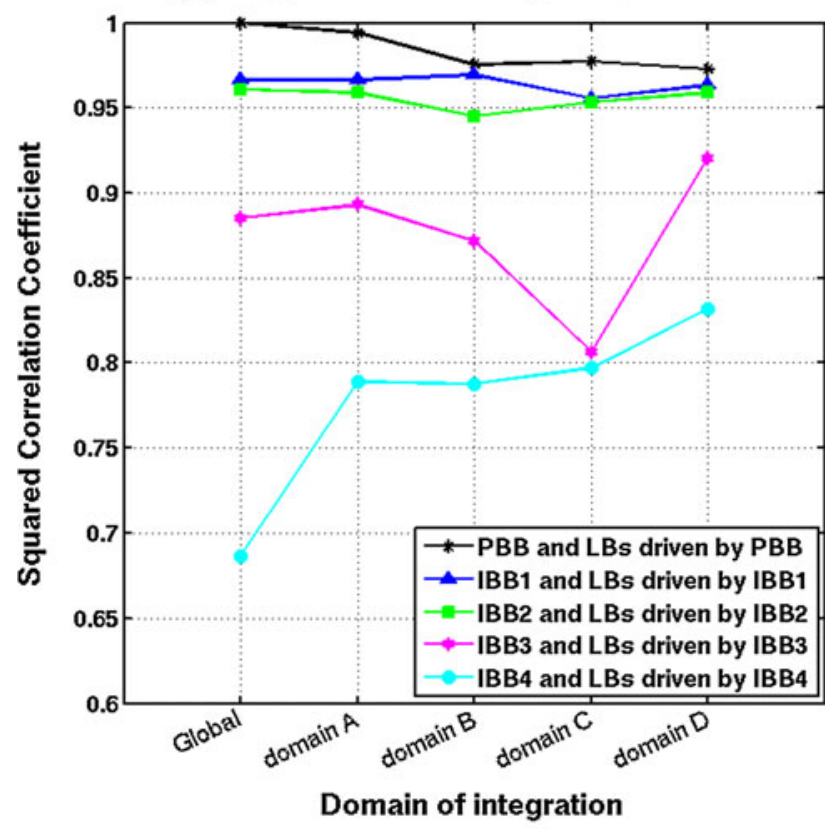

(b) Large-scale transient component

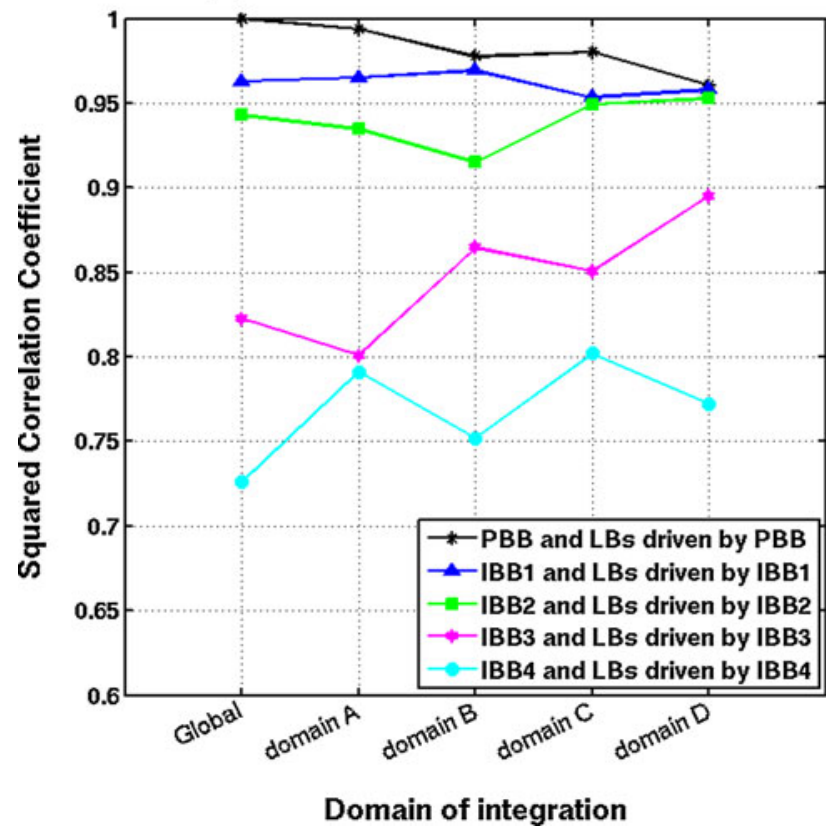

Fig. $14 \mathrm{LB}$ and BB squared correlation coefficient for a the stationary component and $\mathbf{b}$ the intra-seasonal transient component of largescale precipitation rate, function of the domain of integration

the differences between the four LBs driven by PBB, and the PBB field; these differences reflect nesting errors as discussed previously. The precipitation exhibit a maximum located along the northern West Coast (with values up to $8.9 \mathrm{~mm} /$ day in the PPB), dry conditions in the central continent with less than $2 \mathrm{~mm} /$ day, and a maximum over the Southeastern United States (with values up to
$4.9 \mathrm{~mm} /$ day in the PBB). The four PBB-driven LBs reproduce well these patterns, with just a slight increase of the Alaskan Panhandle maximum in the A, B and C domains. The overall picture shows a very high similarity of LBs precipitation with that of the PBB, with spatial correlation coefficients greater than 0.98 . Similarly a great similarity is also found for the intra-seasonal transient components (see Table 3), reflecting that nesting errors for all the tested LB domain sizes are small in the statistics of the wintertime precipitations.

Figure 5 presents the differences between the large scale of the mean winter precipitation fields simulated by PBB and the four LBs driven by ERA-Interim reanalyses. The four LBs reproduce fairly well the overall precipitation pattern of the PBB, although some differences are noted for the maximum in the Southeastern USA. The corresponding differences for the four IBBs and their driven LBs are presented in Figs. 6, 7, 8 and 9, together with the driving IBB field. The IBB1 simulation (Fig. 6) presents a narrower maximum on the West Coast, and this bias worsens with coarser resolution IBBs: $6.3 \mathrm{~mm} /$ day in IBB2 (Fig. 7), $5.7 \mathrm{~mm} /$ day in IBB3 (Fig. 8) and only $5.4 \mathrm{~mm} /$ day in IBB4 (Fig. 9). The IBB1, IBB2 and IBB3 simulations present overly weak maximum in the Southeastern USA, and in IBB4, this maximum is shifted very much to the south. All LBs tend to correct the IBB error present in the amplitude of the West Coast maximum.

A similar underestimation of the intra-seasonal transient-eddy precipitation magnitude is also noted in the low-resolution IBBs. An example is presented in Fig. 10, which shows the intra-seasonal transient component of large-scale precipitations for IBB4 and the LBs driven by IBB4, compared to PBB. While the PBB presents values up to $11.3 \mathrm{~mm} /$ day in the West Coast, the IBB4 shows a maximum of only $7.7 \mathrm{~mm} /$ day, and its Southeastern USA maximum is substantially displaced to the south. As was the case for the mean precipitation, the LBs tend to recover the PBB West Coast maximum. However, in general, they have difficulty correcting the Southeastern USA maximum when it is displaced to the south (Figs. 9, 10).

In order to quantify the IBB (and LB) errors relative to the PBB fields, we use Taylor diagrams (Taylor 2001) that present simultaneously three statistics:

- The spatial correlation coefficient (radial lines):

$R_{i}=\frac{\left\langle\left(\Psi_{i}-\left\langle\Psi_{i}\right\rangle\right)\left(\Psi_{P B B}-\left\langle\Psi_{P B B}\right\rangle\right)\right\rangle}{\sqrt{\left\langle\left(\Psi_{i}-\left\langle\Psi_{i}\right\rangle\right)^{2}\right\rangle} \sqrt{\left\langle\left(\Psi_{P B B}-\left\langle\Psi_{P B B}\right\rangle\right)^{2}\right\rangle}}$,

- The ratio of spatial standard deviations (black dotted circles): 
Fig. 15 Mask used to cover the underground points (the black regions) at different levels
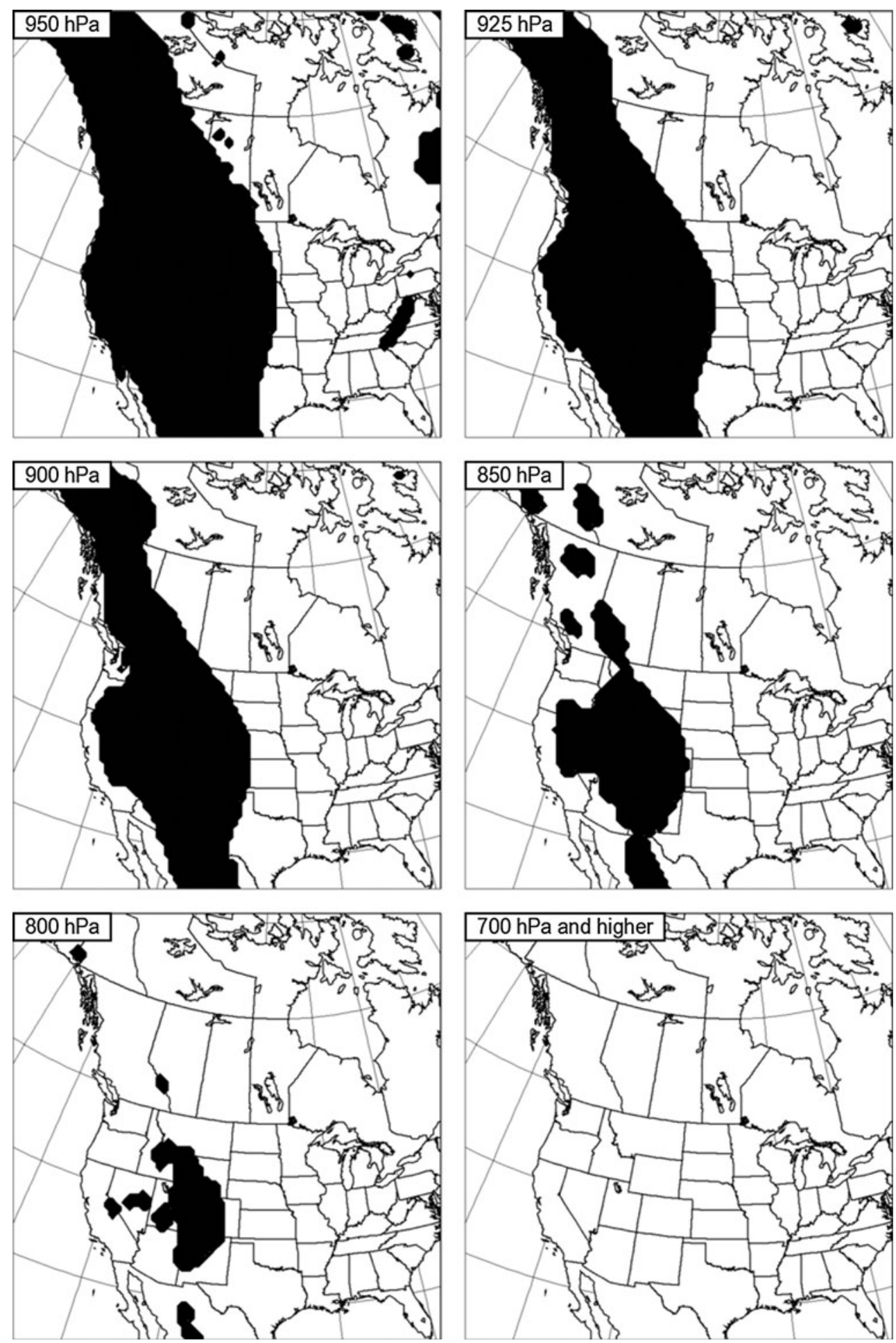

$\Gamma_{i}=\sqrt{\frac{\left\langle\left(\Psi_{i}-\left\langle\Psi_{i}\right\rangle\right)^{2}\right\rangle}{\left\langle\left(\Psi_{P B B}-\left\langle\Psi_{P B B}\right\rangle\right)^{2}\right\rangle}}$,

$$
\begin{aligned}
d_{i} & =\frac{\left\langle\left[\left(\Psi_{i}-\left\langle\Psi_{i}\right\rangle\right)-\left(\Psi_{P B B}-\left\langle\Psi_{P B B}\right\rangle\right)\right]^{2}\right\rangle}{\left\langle\left(\Psi_{P B B}-\left\langle\Psi_{P B B}\right\rangle\right)^{2}\right\rangle} \\
& =1+\Gamma_{i}^{2}-2 \Gamma_{i} R_{i} .
\end{aligned}
$$

- The relative centered mean square difference (blue dashed circles):

The angular brackets $\langle\cdot\rangle$ denote a spatial average over the analysis domain, $i$ represents the IBB or the LB 
Fig. 16 Vertical distribution of RMS errors in the stationary component of large-scale specific humidity $(\mathrm{g} / \mathrm{kg})$ simulated by: a the four LBs driven by PBB, b ERA-Interim and the four LBs driven by ERA-Interim, c IBB1 and the four LBs driven by IBB1, d IBB2 and the four LBs driven by IBB2, e IBB3 and the four LBs driven by IBB3 and f IBB4 and the four LBs driven by IBB4
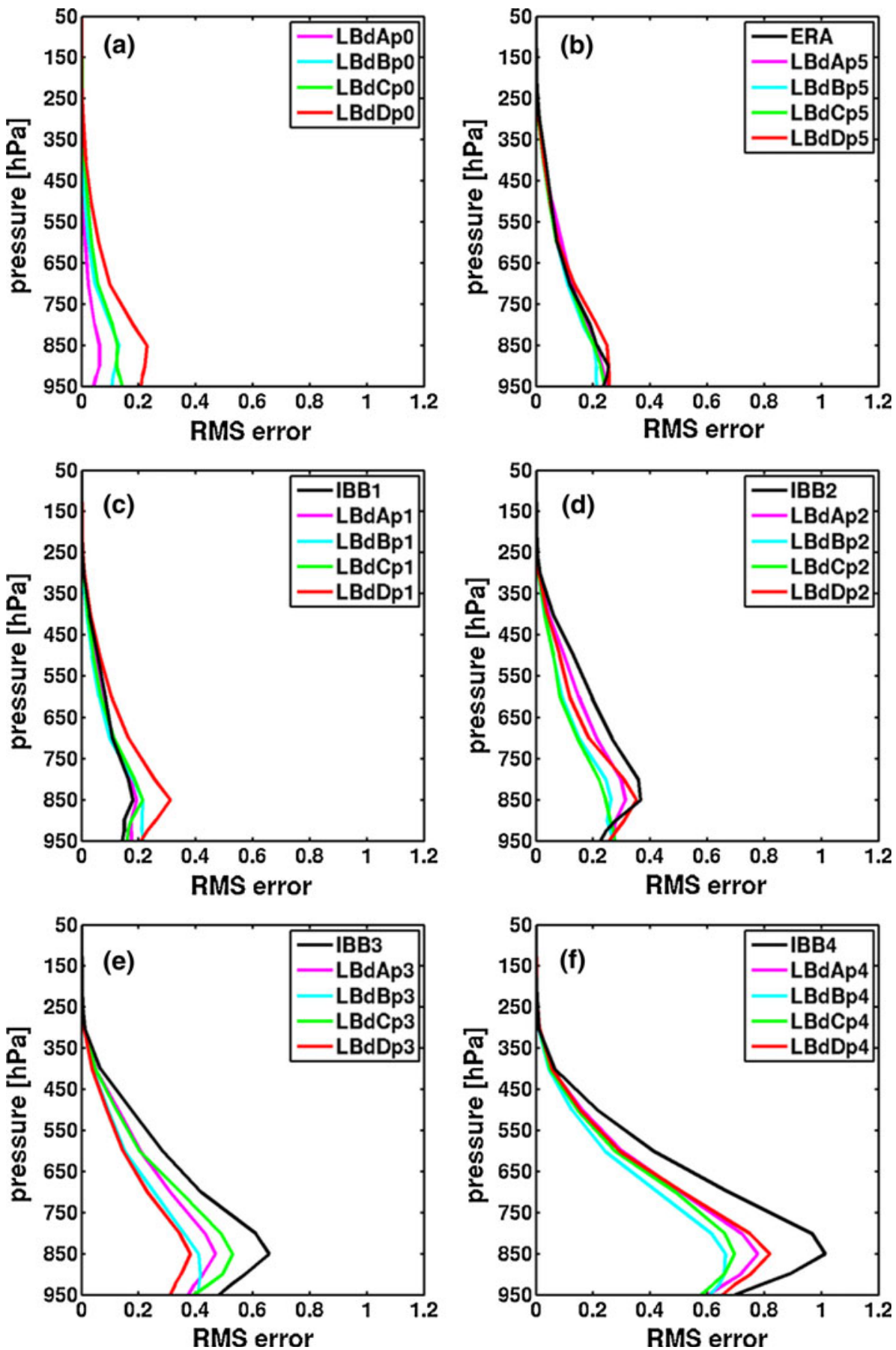

simulations, and $\Psi$ stands for the stationary or intra-seasonal transient components of the field.

On the Taylor diagrams, the scores of the driving fields are plotted in black and those of the LB are shown in colours indicating their domain: magenta for domain $\mathrm{A}$, blue for domain $\mathrm{B}$, green for domain $\mathrm{C}$ and red for domain D. A number besides each point identifies the driving fields: 0 for PBB, 1-4 for IBB1 to IBB4, and 5 for ERAInterim. The PBB, the reference field, is plotted on the origin of the diagram, corresponding to perfect score (zero relative mean square errors, unity spatial correlation coefficient and ratio of spatial standard deviations).

The large-scale precipitation errors in the stationary component are presented in Fig. 11, while the errors corresponding to the intra-seasonal transient component are presented in Fig. 12. The scores of the four IBBs indicate a systematic increase of errors with decreasing resolution, for both the mean and intra-seasonal transient components. The deficient standard deviations of IBBs' precipitation reflect the reduced precipitations on the West Coast in 
Fig. 17 Vertical distribution of RMS errors in the intra-seasonal transient component of largescale specific humidity $(\mathrm{g} / \mathrm{kg})$ simulated by: a the four LBs driven by PBB, b ERA-Interim and the four LBs driven by ERA-Interim, c IBB1 and the four LBs driven by IBB1, d IBB2 and the four LBs driven by IBB2, e IBB3 and the four LBs driven by IBB3 and f IBB4 and the four LBs driven by IBB4
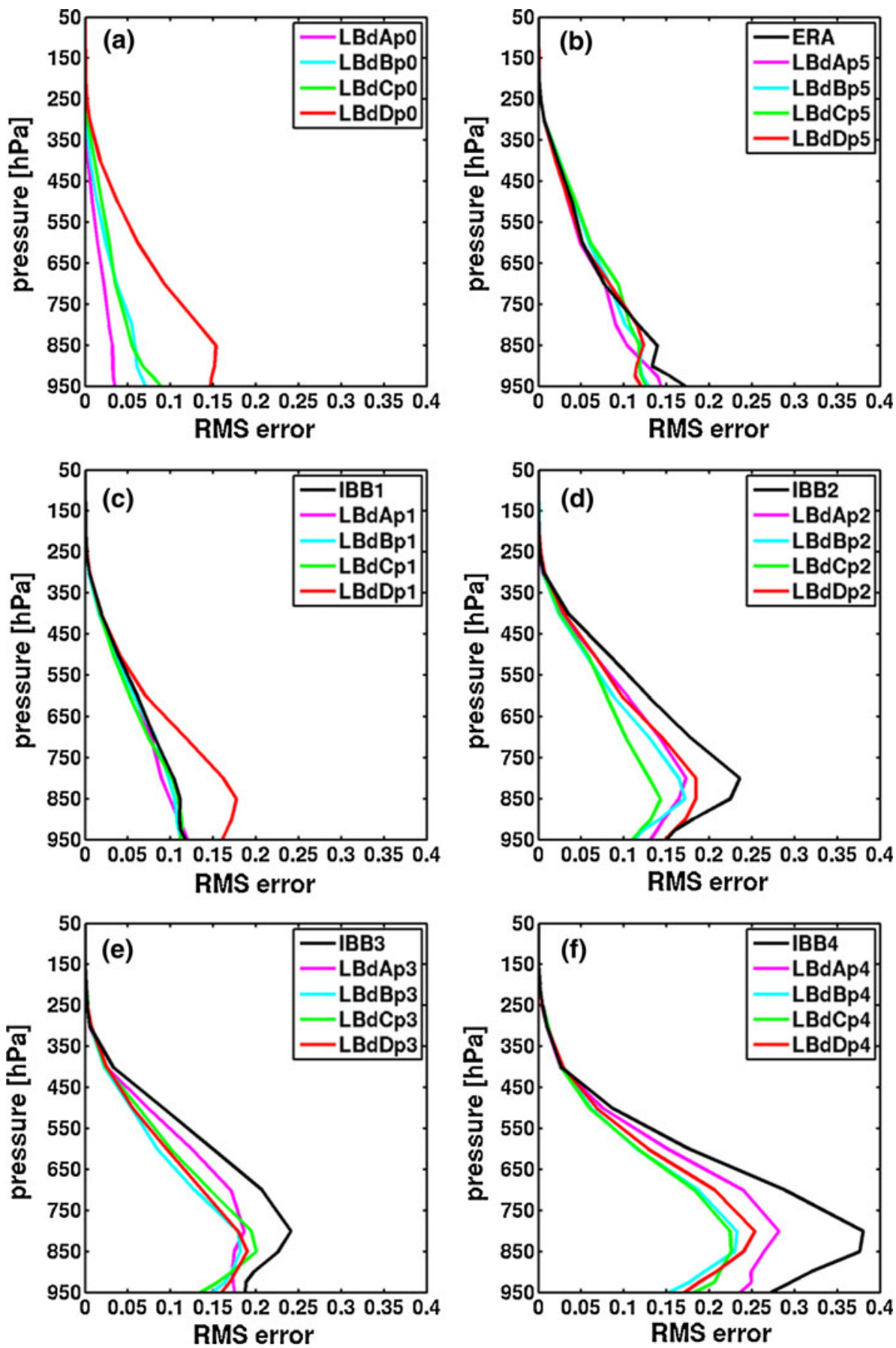

low-resolution simulations compared to the PBB. We have seen before that all LBs tend to correct this error; in the Taylor diagrams, this is seen by points approaching the red line of unitary ratio of spatial standard deviations. For a given set of LBC, the best scores, for both the mean and intra-seasonal transient components, are generally obtained with LBs over the largest domain D (red points), and the LB scores improve with increasing resolution of the driving model. The better scores of the LB compared to their IBB arise from the better-resolved Rocky Mountains topography that improves the simulated precipitation in LBs compared to their coarse-resolution driving fields. On the other hand we saw before that the LBs have little skill in correcting the errors in the Southeastern USA where coarse-resolution IBB showed a southward shift of the maximum precipitation.

A direct way to see if there is a reduction of the driving model errors in the LB simulations is to plot the domainaverage LB errors as a function of the corresponding driving model errors. This is presented in Fig. 13 for the 
(a) Large-scale stationary component

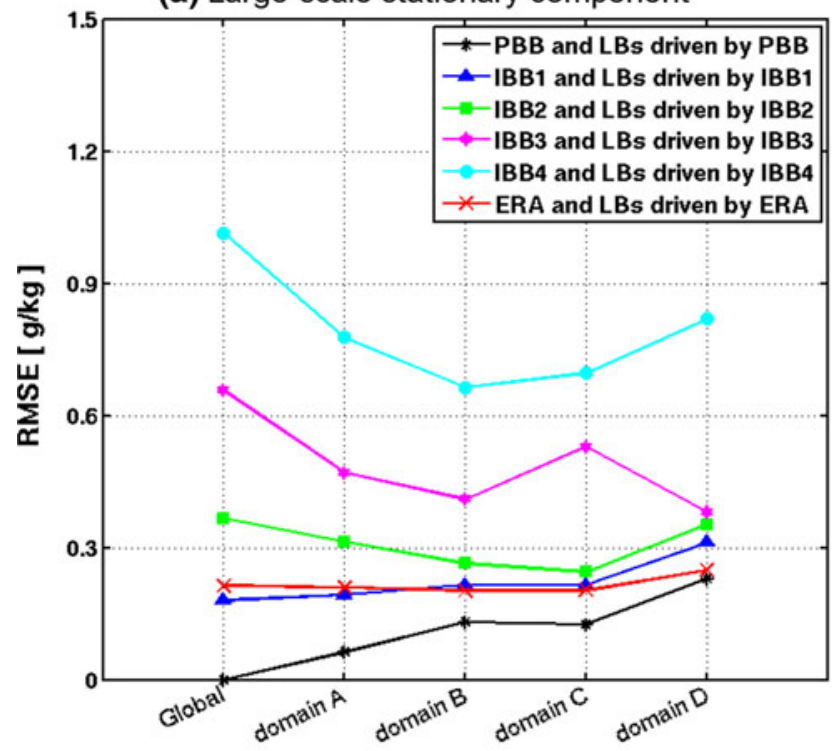

Domain of integration

(c) Large-scale transient component

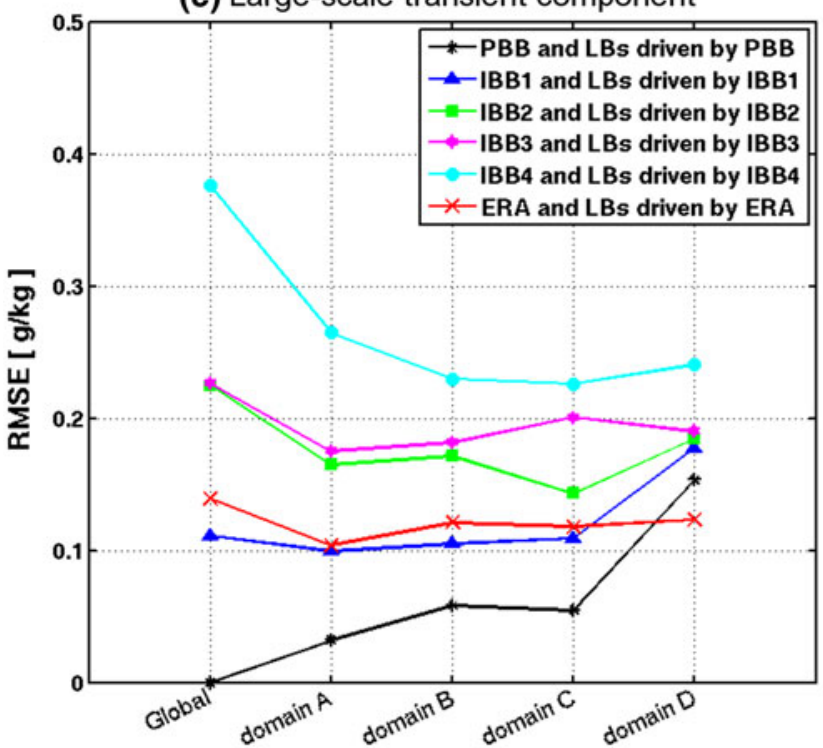

Domain of integration (b) Large-scale stationary component

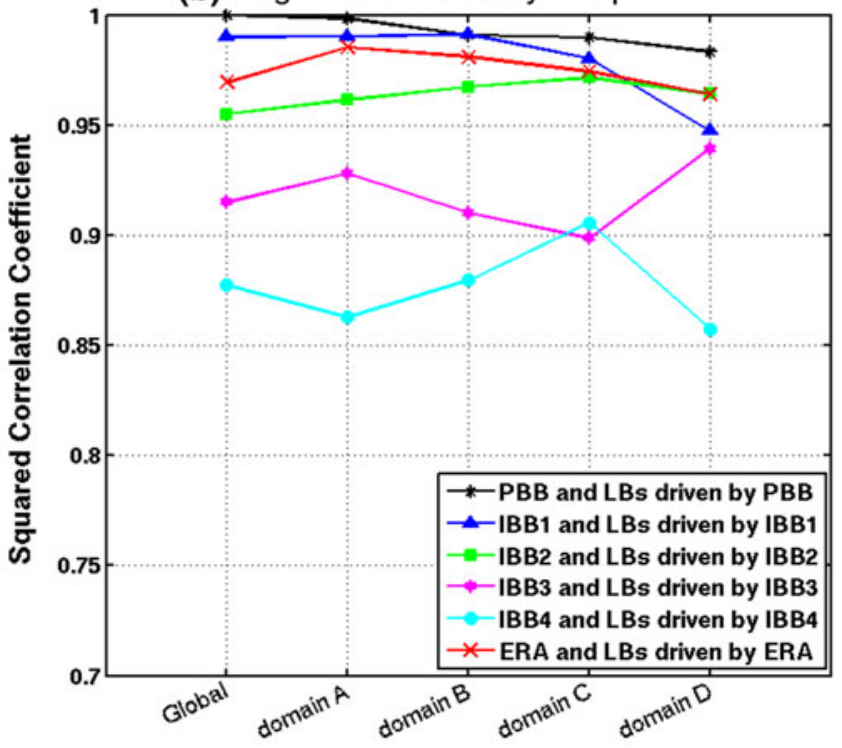

Domain of integration

(d) Large-scale transient component

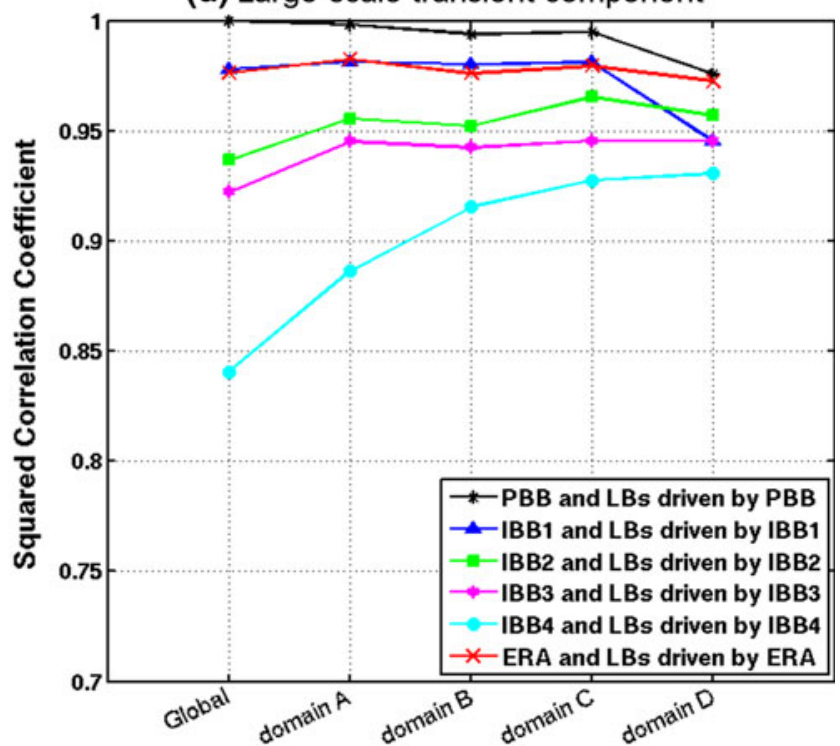

Domain of integration

Fig. $18 \mathrm{LB}$ and BB RMS errors and squared correlation coefficients for $\mathbf{a}, \mathbf{b}$ the stationary component and $\mathbf{c}$, $\mathbf{d}$ the intra-seasonal transient component of large-scale specific humidity $(\mathrm{g} / \mathrm{kg})$ at $850 \mathrm{hPa}$, function of the domain of integration

large-scale precipitation. The errors are computed as ratio of spatial standard deviations (Eq. 5) and root mean square (RMS) errors from the PBB field:

$\operatorname{RMSE}_{P B B}=\sqrt{\left\langle\left(\Psi_{i}-\Psi_{P B B}\right)^{2}\right\rangle}$,

where the brackets $\langle$.$\rangle denotes a spatial average over the$ analysis domain, $i$ represents the IBB or the LB, and $\Psi$ stands for the time mean or the intra-seasonal transient components. The upper panels correspond to the time mean, while the lower panels correspond to the intra-seasonal transient component. A diagonal black line indicates where the LB errors would be equal to the driving $\mathrm{BB}$ model errors.

For the RMS errors (panels a and $b$ ), points located below the diagonal line correspond to smaller errors in the LB simulations than in the corresponding driving model, showing that there is an overall reduction of errors of the driving model 
Fig. 19 Vertical distribution of RMS errors in the stationary component of large-scale zonal wind (knots) simulated by: a the four LBs driven by PBB, b ERA-Interim and the four LBs driven by ERA-Interim, c IBB1 and the four LBs driven by IBB1, $\mathbf{d}$ IBB2 and the four LBs driven by IBB2, e IBB3 and the four LBs driven by IBB3 and f IBB4 and the four LBs driven by IBB4
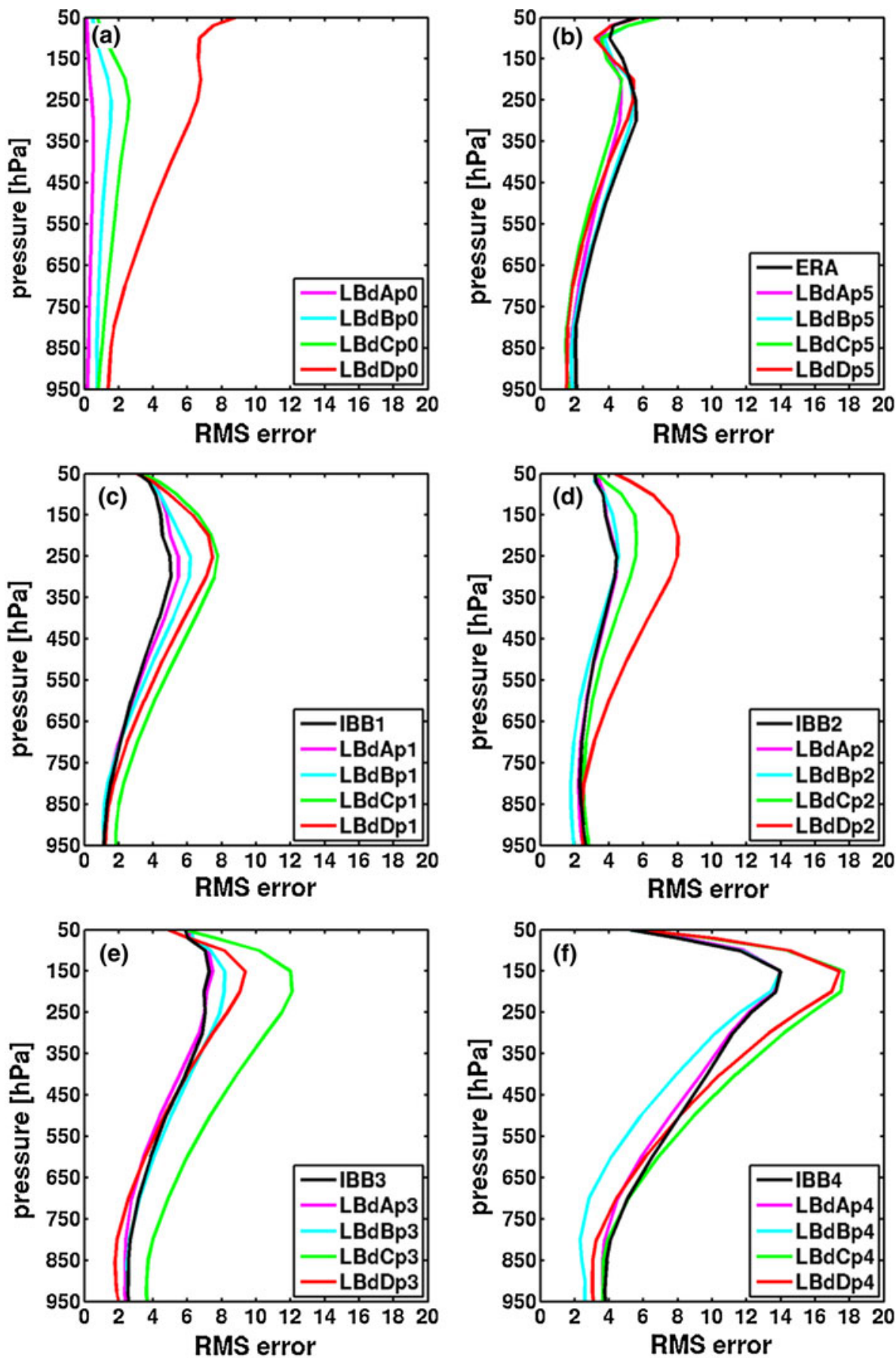

in the LB simulations, while points located above the diagonal line indicate that the LB simulations have larger errors than their driving model, therefore increase the errors.

For the normalized STD (panels $\mathrm{c}$ and d), the red dashed line indicates a spatial STD equal to the reference field STD, points with normalized STD greater than 1 exceed the reference spatial variance and points with normalized STD smaller than 1 have a smaller spatial variance than the reference field. Thus, LBs lying on the red line have spatial variations of the correct amplitude, while LBs situated on the black diagonal line have the same spatial variations as their driving model.

Figure 13 shows that LB nesting errors (i.e. the errors of LBs that are driven by $\mathrm{PBB}$ ), represented by asterisks, are small: around $0.4 \mathrm{~mm} /$ day for the RMS error of time mean (panel a) and up to $0.66 \mathrm{~mm} /$ day for the RMS error of transient component (panel b). The spatial STD shows a small tendency of the LB to have somewhat excessive 
Fig. 20 Vertical distribution of RMS errors in the intra-seasonal transient component of largescale zonal wind [knots] simulated by: a the four LBs driven by PBB, b ERA-Interim and the four LBs driven by ERA-Interim, c IBB1 and the four LBs driven by IBB1, d IBB2 and the four LBs driven by IBB2, e IBB3 and the four LBs driven by IBB3 and f IBB4 and the four LBs driven by IBB4
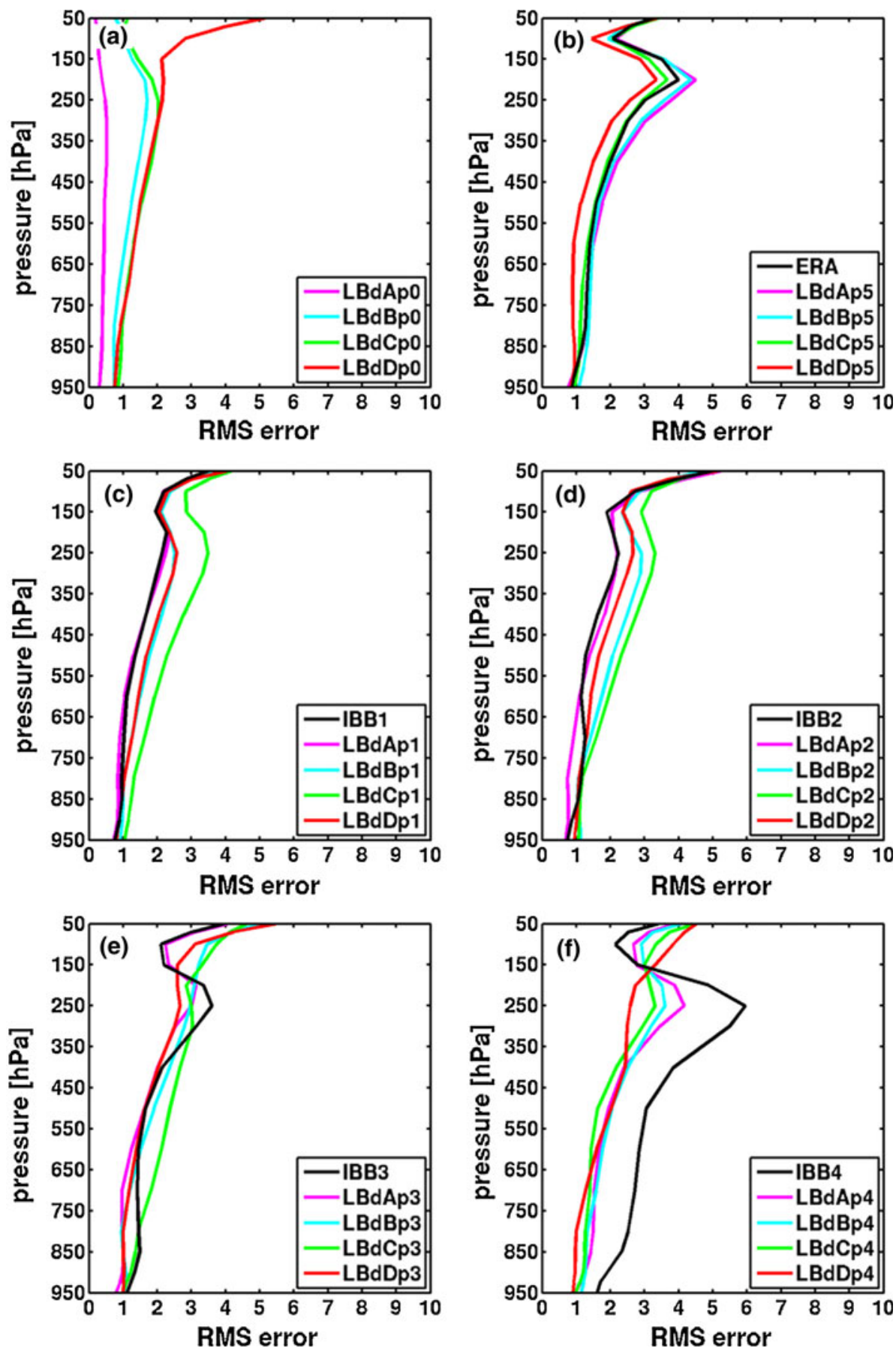

spatial STD in the stationary part of the field (panel c), although the transient component is nearly perfect (panel d). It is noteworthy that the PBB-driven LB RMS errors in the time mean are almost insensitive to the LB domain, while for the intra-seasonal transient component the RMS errors grow somewhat with LB domain size, although they remain small. The opposite is noted for the spatial STD, where the largest domain is the closest to the PBB. For IBB-driven LBs, their RMS errors remain virtually unchanged, equal to the nesting error value, so long as
IBBs exhibit small errors (see the LBs driven by IBB1 in panels $a$ and $b$, and by IBB2 in panel $a$, that have the same error level as the LBs driven by PBB), while LBs driven by IBBs with larger RMS errors exhibit larger errors. Nevertheless, most LB RMS errors are below the diagonal line, indicating an overall reduction of the IBB errors. As mentioned previously, the most important feature of IBB errors consist in a very small ratio of standard deviation indicating an important loss in the patterns' amplitude. Figure 13c, d show that all LBs have a better STD then 
(a) 250-hPa stationary component

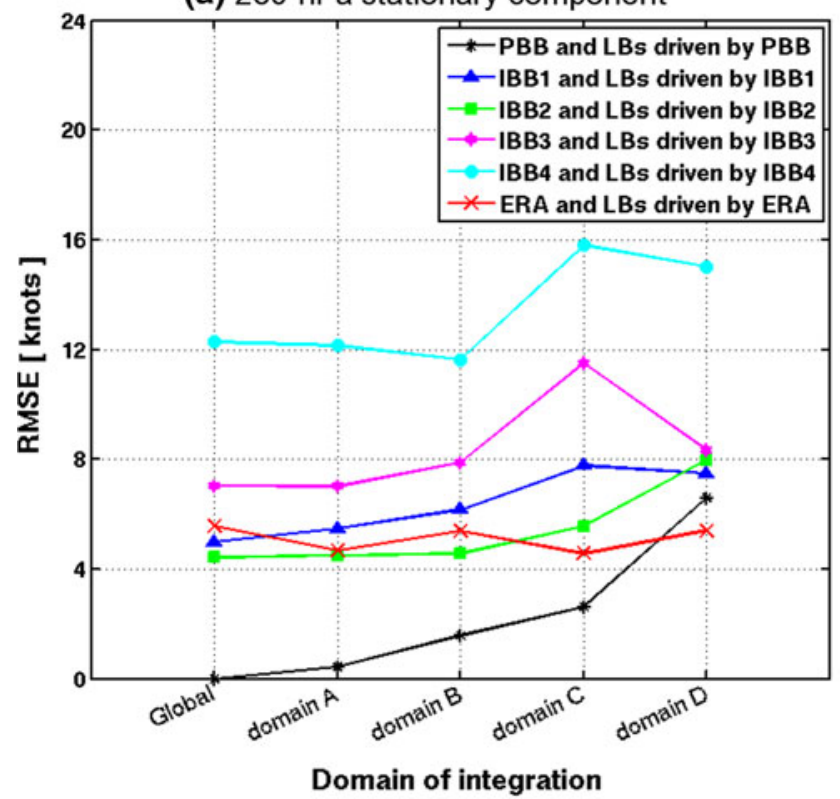

(c) $850-\mathrm{hPa}$ stationary component

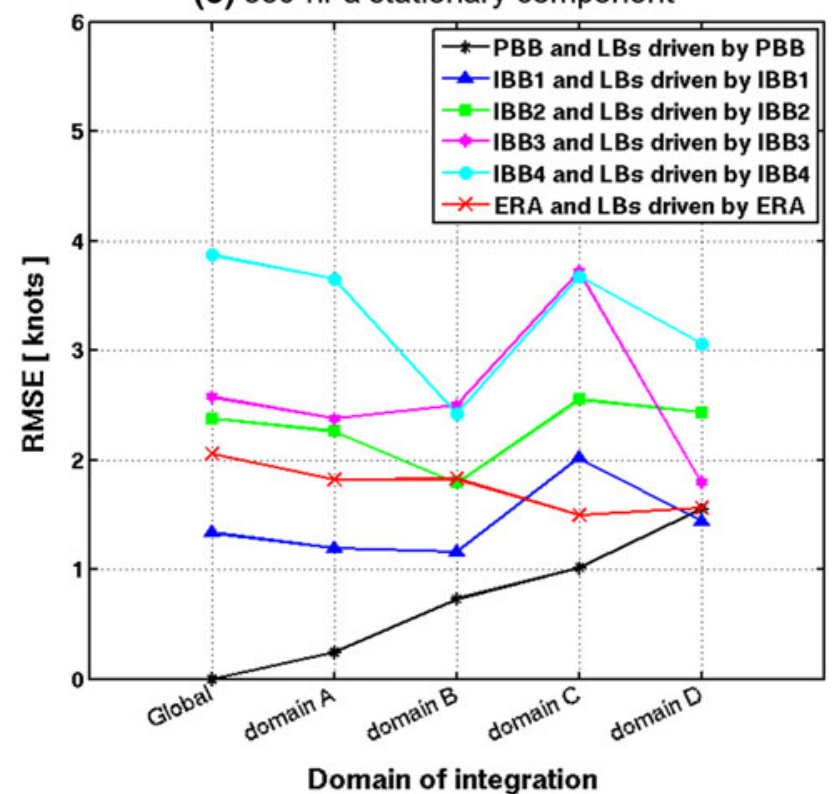

(b) 250-hPa transient component

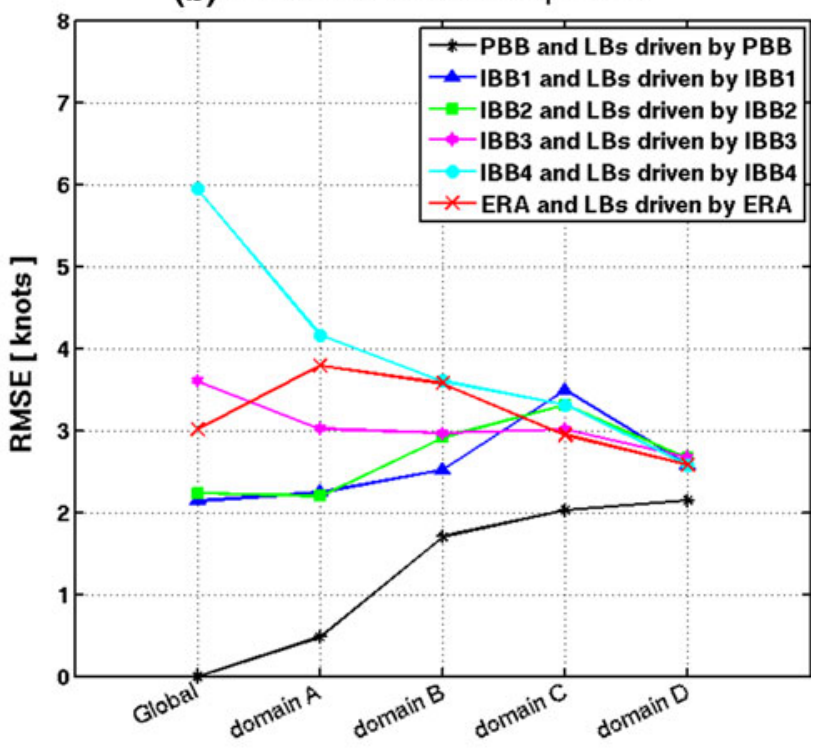

Domain of integration

(d) $850-\mathrm{hPa}$ transient component

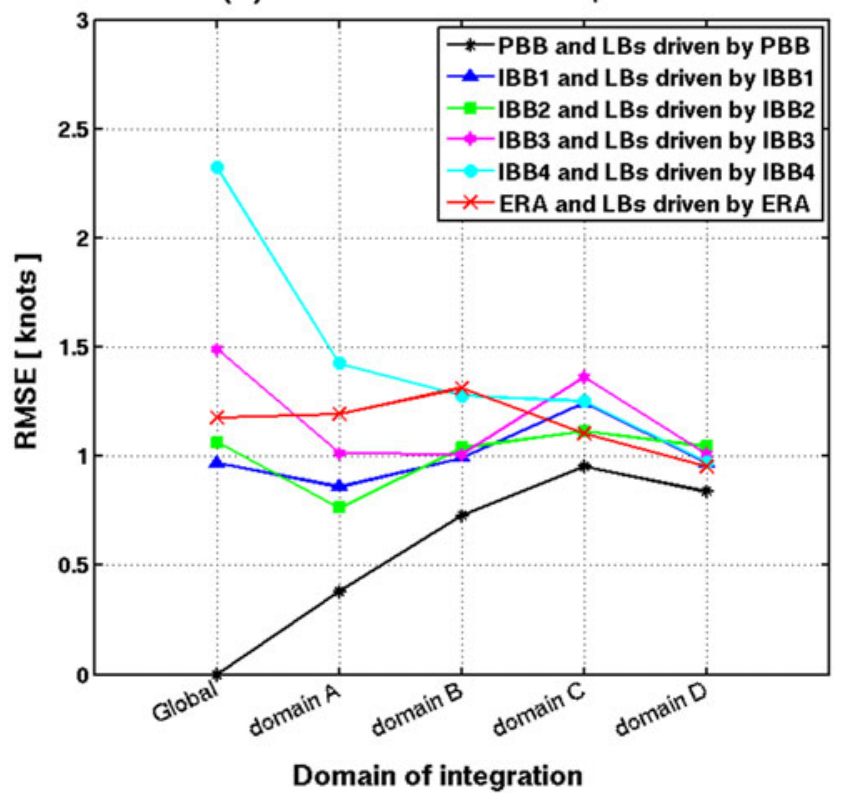

Fig. 21 LB and BB RMS errors for a, $\mathbf{c}$ the stationary component and $\mathbf{b}$, $\mathbf{d}$ the intra-seasonal transient component of large-scale zonal wind (knots) at 850 and $250 \mathrm{hPa}$, function of the domain of integration

their driving IBBs, approaching the right patterns' amplitude. The overall picture shows that the LBs over the largest domain (D) have the best ratio of standard deviation in both the stationary and the transient component, and also the smallest RMS errors in the stationary component.

Figure 14 presents the squared correlation coefficient as a function of the domain of integration, with the PBB and IBB indicated as "Global". The error of PBB-driven LBs increases somewhat with larger domain sizes, but the correlation remains quite large. On the other hand, the errors of LB simulations driven by coarse-resolution IBB decrease with the use of larger domains, the squared correlation coefficient increasing by values ranging from 5 to $15 \%$ above the value of the IBB, thus indicating an improvement in the large scales compared to the driving LBC. LB simulations driven by intermediate-resolution IBBs are rather insensitive to domain size, which is comforting given the arbitrariness of the choice of domain size. 

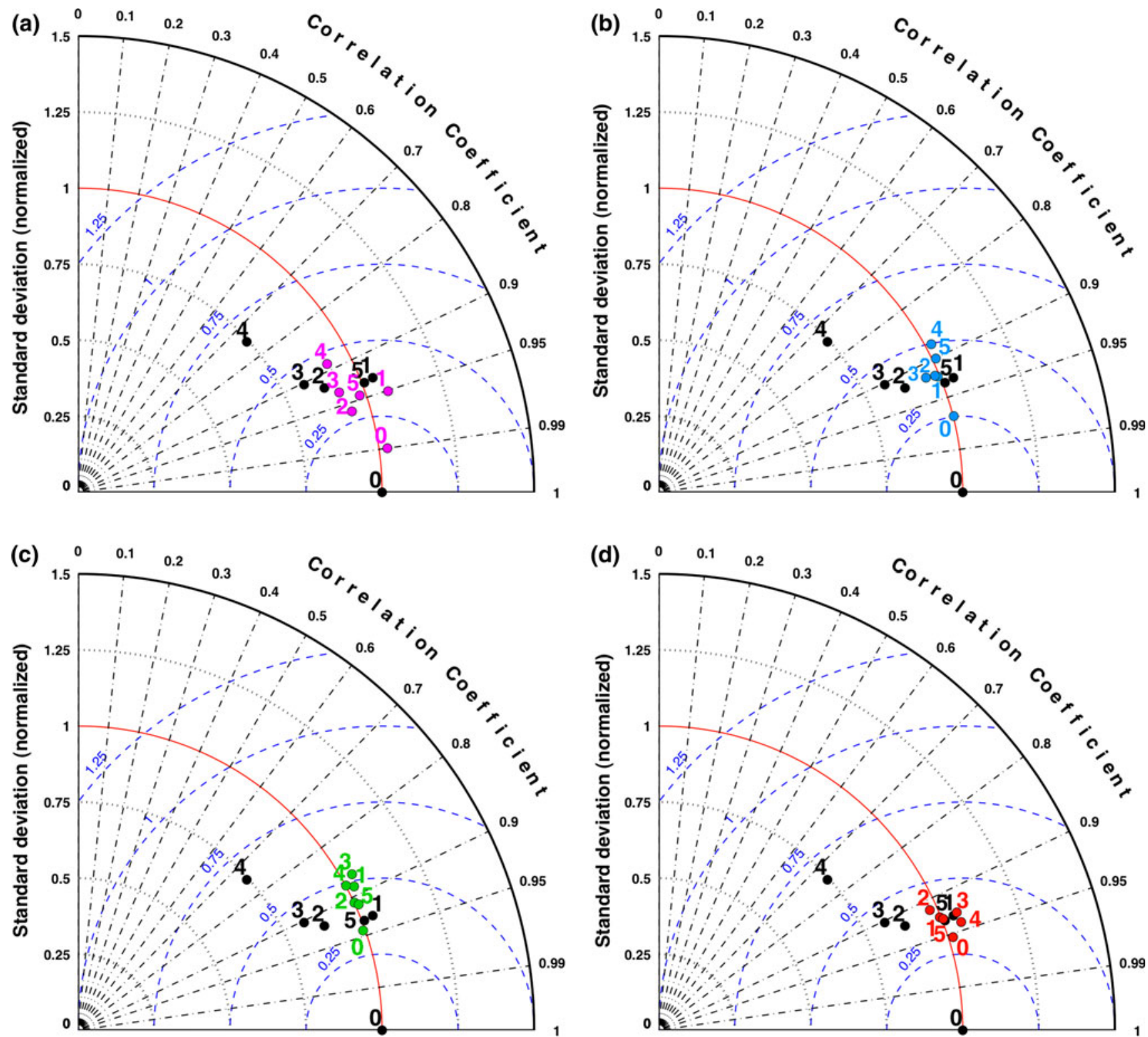

LBs domain A LBs domain C
LBs domain B

Fig. 22 Taylor diagrams showing the large-scale zonal wind errors at $850 \mathrm{hPa}$ in the intra-seasonal transient component of IBBs, ERA and LBs

We can summarise our results so far as follows. The nesting error value provides a measure of the minimum unavoidable error associated with the nesting technique under ideal conditions of perfect LBC and a perfect model; the errors can only be larger when an RCM is driven by imperfect LBC or for an imperfect model. Large regional domains can afford an improvement of the large-scale precipitation field compared to the driving model. The improvement however is rather modest given the important increase in computational cost, and the errors increase as the LBC degrade.

\subsection{Large-scale specific humidity}

We now turn our attention to the atmospheric water vapour field. In order to reduce the volume of information, we will present vertical profiles of scores averaged on pressure levels. Underground grid points have been removed following the masking procedure of Boer (1982). A single mask has been designed to remove all points laying underground in any one of the PBB, IBBs, ERA-Interim and LBs datasets after their interpolation on domain A (Fig. 15). 
Fig. 23 LB versus BB RMS errors and squared correlation coefficients for $\mathbf{a}, \mathbf{b}$ the stationary component and c, $\mathbf{d}$ the intra-seasonal transient component of small-scale precipitation. The asterisks stand for LBs nested by PBB, the triangles for LBs nested by IBB1, the squares for LBs nested by IBB2, the stars for LBs nested by IBB3 and the circles for LBs nested by IBB4 (a) Small-scale stationary component

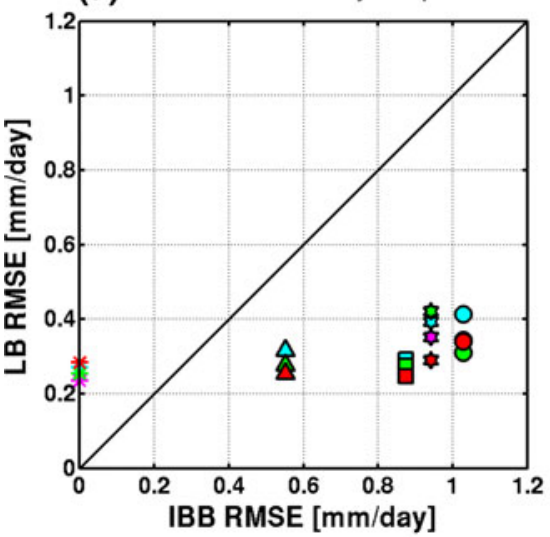

(c) Small-scale transient component

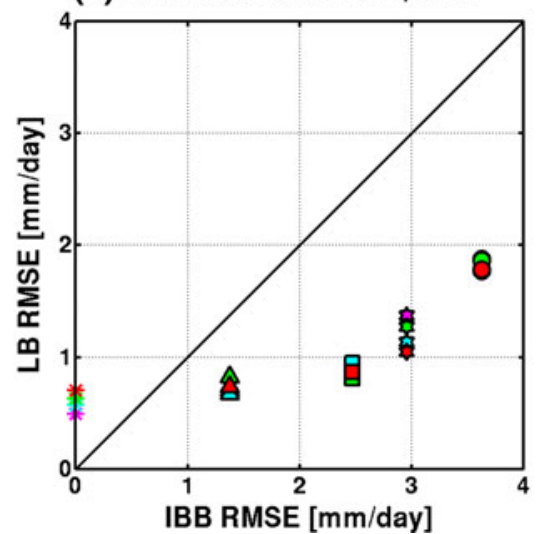

(b) Small-scale stationary component

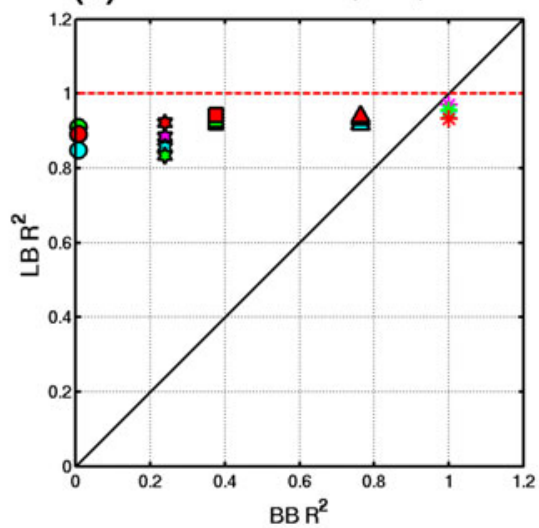

(d) Small-scale transient component

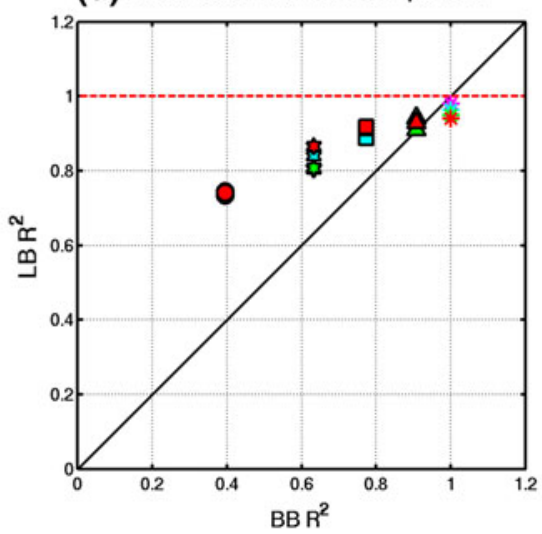

* LBdAp0

$\triangle$ LBdAp1

LBdAp2

LBdAp3

LBdAp4

$X$ LBdAp5

* LBdBp0

$\triangle$ LBdBp1

LBdBp2

L LBdBp3

LBdBp4

X LBdBp5

* LBdCp0

$\triangle$ LBdCp1

LBdCp2

LBdCp3

LBdCp4

$X$ LBdCp5

* LBdDp0

$\triangle$ LBdDp1

LBdDp2

LBdDp3

LBdDp4

X LBdDp5
The vertical profiles of specific humidity RMS errors are presented in Fig. 16 for the time mean and in Fig. 17 for the intra-seasonal transient component. Panels 16a and 17a present the PBB-driven LBs errors. We note again that the nesting errors generally increase with LAM domain size, with the maximum in low levels. Panels $16 \mathrm{~b}$ and $17 \mathrm{~b}$ present the errors of LB simulations driven by ERAInterim. We note that the LB errors are relatively insensitive to domain size this time. The black line gives the apparent error of ERA-Interim when compared to the PBB used as reference, which is of course equivalent to the error of the PBB compared to ERA-Interim. This error is similar to the nesting errors on the larger domain D (panels 16a and 17a); this makes sense, as a LAM operating on increasingly larger domains should asymptotically behave as a global model. Panels c-f in Figs. 16 and 17 give the errors of LBs and their driving IBB simulations. The IBB1 error is roughly of the same order of magnitude as ERAInterim apparent error, and the error of the other IBB increases substantially with coarser resolution. For all LB simulations driven by IBB $n$ with $n>1$, the nested model simulations succeed in reducing the driving-model errors by roughly a factor of two.

Figure 18 presents an overall picture of LB and BB error in the large scales of the $850-\mathrm{hPa}$ specific humidity, by plotting the RMS error and squared correlation coefficient as a function of the domain of integration. We note that the nesting errors (i.e. the errors of the LBs driven by the PBB, represented in black) increase with LB domain size, but remain small (squared correlation coefficients higher than 0.95). Generally LB simulations driven by coarse-resolution IBBs reduce the RMS error and increase the correlation coefficient compared to the driving model. There is however no systematic relationship between the LBs errors and their domain sizes.

\subsection{Large-scale zonal wind component}

The vertical profiles of RMS errors for large-scale zonal wind are presented in Fig. 19 for the time mean and in Fig. 20 for the intra-seasonal transient component. As for specific humidity, the underground grid points have been removed using the mask shown in Fig. 15. Figures 19a and 20a present the RMS errors of LBs simulations driven by the PBB, and hence define nesting errors. As for specific humidity, nesting errors increase with LB domain size, but remain relatively small, except with the largest domain D. Panels 19b and 20b show the errors of LB simulations driven by ERA-Interim. We note that the ERA-Interim driven LB zonal wind errors are relatively 
Fig. 24 LB versus BB RMS errors and squared correlation coefficients for $\mathbf{a}, \mathbf{b}$ the stationary component and $\mathbf{c}, \mathbf{d}$ the intra-seasonal transient component of small-scale zonal wind at $850 \mathrm{hPa}$. The asterisks stand for LBs nested by PBB, the triangles for LBs nested by IBB1, the squares for LBs nested by IBB2, the stars for LBs nested by IBB3 and the circles for LBs nested by IBB4 (a) Small-scale stationary component

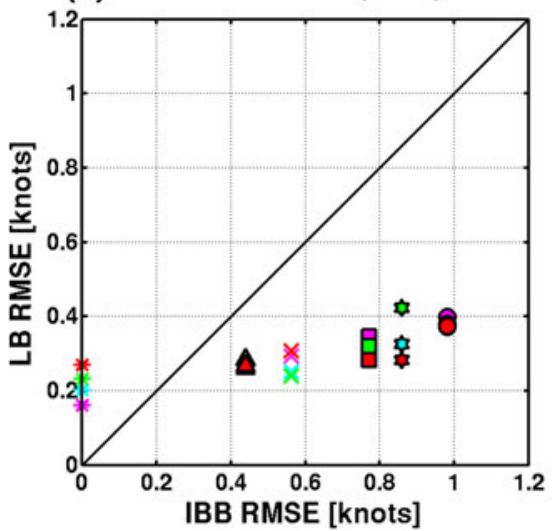

(c) Small-scale transient component

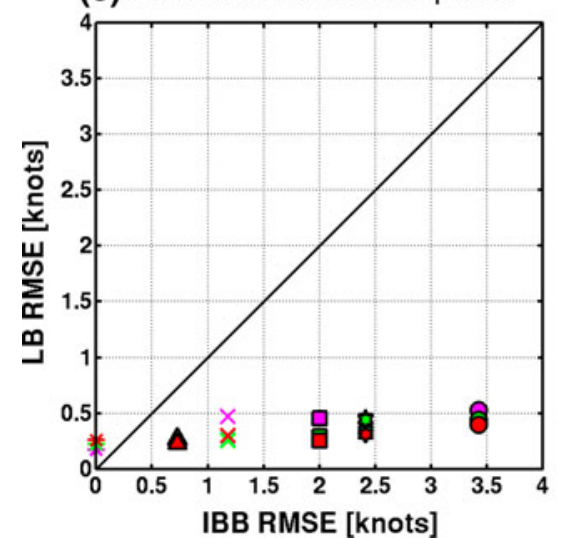

(b) Small-scale stationary component

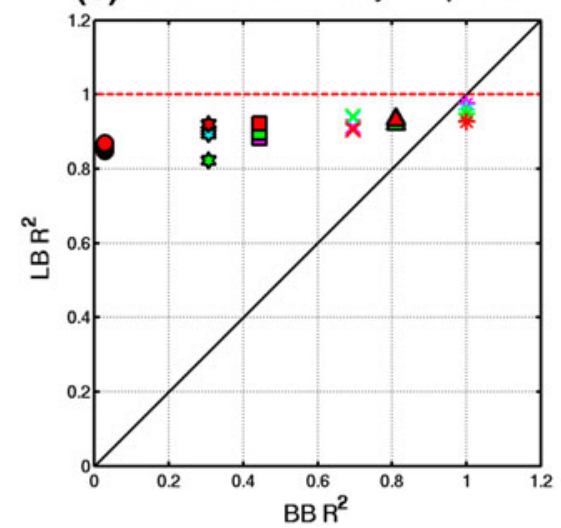

(d) Small-scale transient component

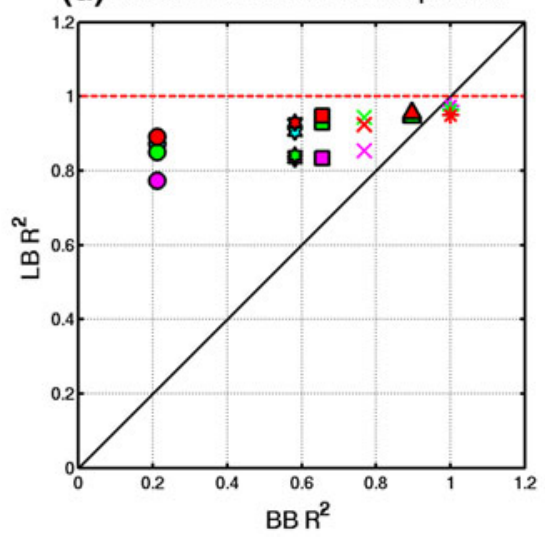

* LBdAp0

$\triangle$ LBdAp1

LBdAp2

LBdAp3

LBdAp4

$X$ LBdAp5

* LBdBp0

$\triangle$ LBdBp1

$\square$ LBdBp2

5 LBdBp3

LBdBp4

$X$ LBdBp5

* LBdCp0

$\triangle$ LBdCp1

LBdCp2

LBdCp3

LBdCp4

$\mathrm{X}$ LBdCp5

* LBdDp0

$\triangle$ LBdDp1

LBdDp2

LBdDp3

LBdDp4

X LBdDp5 insensitive to domain size, as was the case for specific humidity. The black line gives the apparent error of ERAInterim when compared to the PBB used as reference; this error is of the same order as, although somewhat smaller than, the nesting errors on the larger domain D (panels 19a and 20a), indicating again that a nested model operating on increasingly larger domains behave similarly to a global model. IBB1 (Figs. 19c, 20c) and IBB2 (Figs. 19d, 20d) exhibit errors of the same order of magnitude as the apparent errors of ERA-Interim; the LBs driven by these IBBs increase slightly the errors. On the other hand, IBB3 (Figs. 19e, 20e) and IBB4 (Figs. 19f, 20f) have errors that are larger than the apparent errors of ERA-Interim. The LBs driven by these IBBs exhibit errors of mixed character, with some reduction at low levels in both the time mean and the transient components; at the jet-stream level, the LB errors are larger than those of the driving models in the stationary component, but they are smaller in the transient component.

The different behaviour at low and high levels is best seen in Fig. 21 showing the LB and BB RMS errors as a function of the domain of integration, for large-scale zonal winds at $250 \mathrm{hPa}$ (upper panels) and at $850 \mathrm{hPa}$ (lower panels). The left-side panels show the errors in the time mean, while the transient component is presented in the right-side panels. For the time mean at $250 \mathrm{hPa}$ (Fig. 21a), most LB simulations increase the errors above the IBB value, by an amount of the order of the nesting errors. At $850 \mathrm{hPa}$ (Fig. 21c), on the other hand, there is no clear systematic variation of LB errors with domain size. For the transient components (Figs. 21b, d), IBB1 and IBB2 errors are in general small and LBs driven by them show a slight increase in the errors when they are run over the larger domains $\mathrm{C}$ and $\mathrm{D}$. On the other hand, coarsest resolution IBB errors are reduced in all LB simulations, the most impressive reduction occurring with the largest domain D. In fact, the set-D LB errors seem rather insensitive to IBB errors, the overall picture showing a convergence toward the error value of the LB driven by perfect LBCs.

As in the case of precipitation, the low-resolution IBB transient components at $850 \mathrm{hPa}$ are also characterised by a small spatial standard deviation, as can be seen in the Taylor diagrams presented in Fig. 22. The improvements brought by the LBs are noted as well in the RMS as in spatial standard deviation, and this for all domains of integrations. The best improvements are obtained with LBs using the largest domain $\mathrm{D}$, where the LBs cluster with spatial correlation coefficients between 0.9 and 0.95 , close to the value of the PBB-driven LB. 

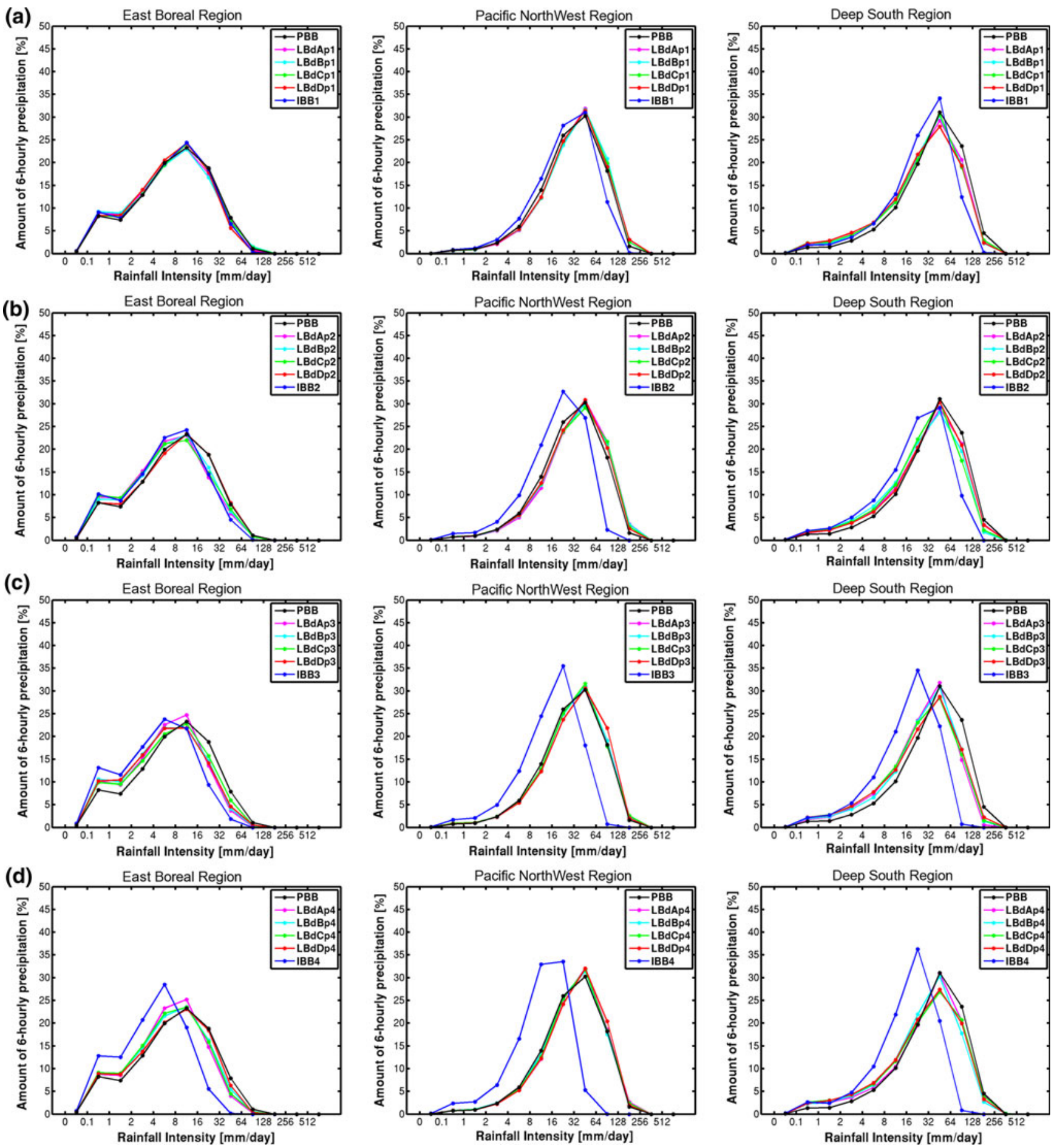

Fig. 25 Total-field 6-hourly precipitation spatial-temporal distributions corresponding to a IBB1 and the LBs driven by IBB1, b IBB2 and the LBs driven by IBB2, c IBB3 and LBs driven by IBB3 and d IBB4 and LBs driven by IBB4. All panels present the reference field

In conclusion, for the large-scale zonal wind simulated by LBs, some reductions of driving-fields errors are noted at low levels in both the stationary and the transient components, while at the upper levels error reductions are seen only in the transient components.
(PBB) in black. The analyses concern three zones: the East Boreal Region (first column), the Pacific North West Region (second column) and the Deep South Region (third column)

\section{The impact of the LBC errors on the LB-simulated small scales and frequency distributions}

Although the main focus of this paper is the study of the impact of LBC errors on the large scales simulated by an 


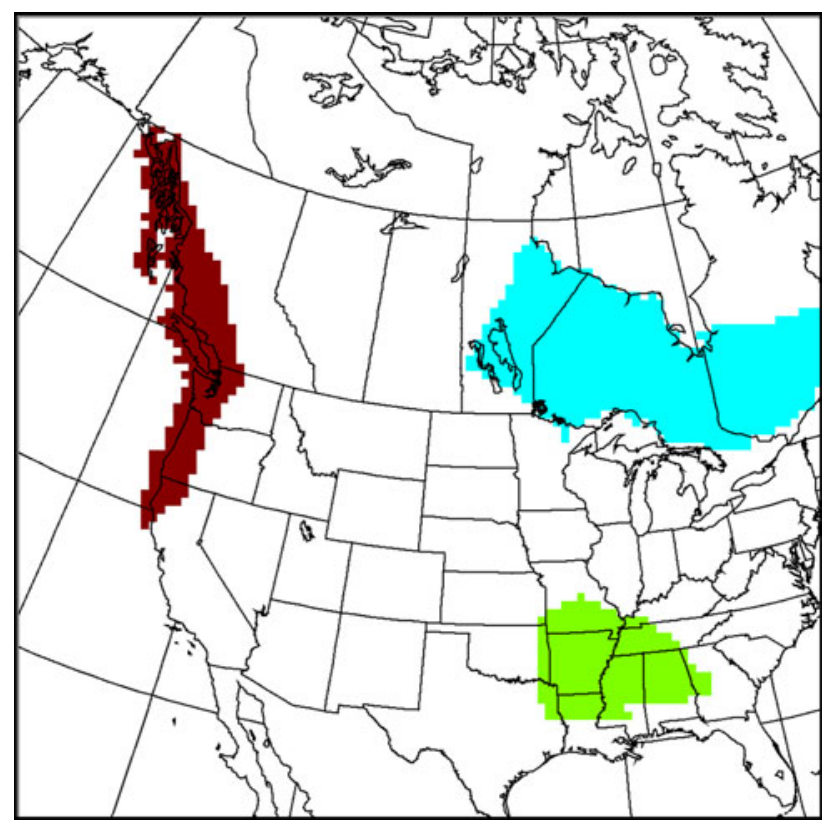

Fig. 26 The three regions used in the precipitation spatial-temporal distribution analyses. The cyan zone represents the East Boreal Region, the red shows to Pacific NorthWest Region and the green color corresponds to the Deep South Region

$\mathrm{RCM}$, it is also of interest to analyze the impact of LBC errors on the small scales developed by an RCM and also on the frequency distribution of variables that constitute the main expectation for added value in dynamical downscaling. The added value of LB small scales is shown in Figs. 23 and 24 that present the LB versus BB RMS errors (Eq. 7) and squared correlation coefficients for the small scales in precipitation and $850-\mathrm{hPa}$ zonal wind fields. The small scales are computed as the difference between the total field and the filtered large scales, as defined earlier. The diagonal black line indicates where the LB scores would be equal to the driving BB scores.

For the RMS errors (right-hand side panels), points located below the diagonal line shows an overall reduction of driving-model errors in the LB simulations, while points located above the diagonal line show that the LB simulations have larger errors than their driving model. The squared correlation coefficient (or the coefficient of determination) is a statistical measure often used in evaluating the strength of a relationship between two variables. In our case, the different points in the left-hand side panels will show in which proportion the spatial variance of the smallscale PBB field is shared by the spatial variance of the small-scale LB fields. The diagonal black line in the leftside panes indicates the proportion in which the spatial variance of the PBB small-scale field is shared by the small-scale driving-model field. Fields with squared correlation coefficient smaller than 0.3 are considered to have no relationship, while fields with squared correlation coefficient greater than 0.7 are related by a strong relationship. As expected, the coefficients of determination for IBB4 and IBB3 are very small indicating a loss of small scales in the coarse-resolution IBB fields.

The small-scale errors of PBB-driven LBs define a sort of lower bound for the errors of any IBB-driven LB simulation. The small scales of LBs driven by any IBB are better than those of the driving IBB, which makes sense given that the IBBs are resolution-deficient to properly resolve the small scales. The LB small-scale errors tend to grow as the resolution of the driving IBB coarsens; but for intermediate-resolution IBB, the LB small-scale errors are rather insensitive to IBB errors, all of them presenting coefficients of determination greater than 0.8 . In general LB small-scale errors show little sensitivity to regional domain size. We note however that even the smaller domain is quite large, so spatial spinup (e.g. Leduc and Laprise 2009; Leduc et al. 2011) is not an issue.

It is well known that coarse-resolution models have difficulties in simulating heavy precipitation events, and hence their distributions are systematically shifted toward smaller intensities. An expection of dynamical downscaling is to recover better frequency distributions. The question however is to what extent the LBC errors can impede RCMs ability to improve on distributions. Fig. 25 presents six-hourly precipitation spatial-temporal distributions for PBB (in black), the four IBBs (in blue) and the corresponding LBs ("set A" in magenta, "set B" in blue, "set $\mathrm{C}$ " in green and "set D" in red). The distributions are computed over the 5 winter seasons and over three regions with widely different climatic characteristics selected amongst the 31 regional masks proposed by Bukovsky (2011) for the North American Regional Climate Change Assessment Program (NARCCAP) (see Fig. 26): the East Boreal region, the Pacific NorthWest region and the Deep South region. One can see in Fig. 25 the systematic shift of IBB distributions towards smaller intensities increasing as their resolution degrades. The LB added value is quite evident. All LBs, independently of their domain size, have distributions very similar to the PBB distribution, thus confirming the LB skill at correcting the driving model distribution shift, despite the presence of errors in driving LBC.

\section{Conclusions}

The main objective of this paper was to study the skill of the large scales simulated by a nested Regional Climate Model (RCM). Specifically whether the use of very large domain of integration could allow correcting the largescale errors present in the lateral boundary conditions (LBC). 
The investigation is carried in a perfect-prognosis framework so that the skill of the RCM at reproducing the observed climate is not an issue. The full version of the Imperfect Big-Brother (IBB) protocol is followed, using a global model and a nested regional model that share the exact same formulations, save for their domain of integration. The global model is the climate version of the Canadian Global Environmental Multiscale model (GEMCLIM) and the regional model is a developmental version of the Canadian RCM (CRCM5). The experiment consists in five global and twenty-four regional simulations. First, a high-resolution $\left(0.45^{\circ}\right)$ GEMCLIM simulation, named the Perfect Big Brother (PBB), serves as reference and also provides "perfect" LBC for some RCM simulations. Then four other global simulations are made using coarser horizontal resolutions $\left(0.9^{\circ}, 1.8^{\circ}, 2.25^{\circ}\right.$ and $3.6^{\circ}$ ); these are named the Imperfect Big Brothers (IBBs) and they provide a set of imperfect LBC for other RCM simulations. Finally, twenty-four high-resolution $\left(0.45^{\circ}\right)$ CRCM5 simulations, named the Little Brothers (LBs), are realized using 6 sets of $\mathrm{LBC}$ (the $\mathrm{PBB}$, the 4 IBBs as well as the ERA-Interim reanalyses), over 4 domains centred on the North American continent, with varying domain sizes; domain A has $108 \times 146$ grid points, domain B has $188 \times 126$ grid points, domain $\mathrm{C}$ has $272 \times 126$ grid points, and domain $\mathrm{D}$ has $272 \times 206$ grid points. The simulations covered 20 months (5 times 4 months) and the analysis focused on the 5-year 3-month wintertime statistics for the time average and the average intra-seasonal standard deviation, over an area of North America corresponding to $100 \times 100$ grid points of the $0.45^{\circ}$ CRCM5 . Three variables were investigated: the precipitation rate, the specific humidity and the zonal wind component.

The PBB and the LBs share identical formulation and resolution, save for the domain of integration and nesting procedure in the LB case. The comparison of the PBBdriven LB simulations over four domains with the PBB allows an estimation of "nesting errors", i.e. errors resulting solely from space and time interpolations as well as nesting scheme. It was found that for most variables the nesting errors tend to increase with domain size, but they remain relatively small. For the largest LB domain (domain D), the nesting errors are of the same order of magnitude as the difference between the PBB simulation and Era-Interim.

The four IBBs are designed such as to have horizontal resolutions typical of global models that are usually involved in climate-change projection studies. The IBB simulation errors with respect to the PBB tend to grow with decreasing resolution, and these errors are much larger than the aforementioned LB nesting errors, even for the largest LB domain. IBBs tend to exhibit weak amplitude climatological features as well as shifts in positions compared to the PBB.
The IBB-driven LBs manage to recover part of the pattern amplitudes in both the stationary and the transient components, in part as a result of their better representation of topography. They show however little skill in eliminating large pattern shifts present in some lower resolution IBBs. In some cases, the use of larger domains seems favourable to allow the LBs to correct some deficiencies present in LBC, but this is not the case for all statistics. For precipitation and specific humidity, LBs driven by IBBs that have small errors (of the order of the nesting error described earlier) maintain errors in the same range. When driven by coarser resolution IBB with larger errors, LBs tend to reduce the errors, and for precipitation, the LBs integrated over the largest domain D generally have the best scores. For zonal wind, some reductions of IBB errors are noted at low levels in both the time-mean and the transient components, while at the upper levels the reductions are seen only in the transient components, particularly over the larger domain D. At jet-stream level, the time mean presents in general an increase in errors.

Two main conclusions emerge from this study with respect to large scales simulated by RCMs:

1. If an RCM is driven by a relatively high resolution GCM, such as IBB1, with small errors (i.e. of magnitude similar to the aforementioned nesting errors), no improvement can be expected in the large scales simulated by the RCM. In such case, a small regional domain, such as $\mathrm{A}$, can be a good solution that will restrain the cost of integration. The added value will be solely in the RCM-simulated small scales that are not present in driving GCM fields.

2. If an RCM is driven by a very low resolution GCM, such as IBB3 and IBB4, presenting large errors, then important reduction of errors is sometimes possible using very large domains. High-resolution RCMs appear to have some skill at recovering part of the amplitude deficient patterns, in both the stationary and the transient components, although in our experiments large shifts of patterns remained largely uncorrected.

The generally held view in regional modelling is that a nested RCM performs dynamical downscaling by developing fine-scale structures that are consistent with the large-scale flow that drives the lateral boundaries. The potential added value of an RCM is expected to be found in the fine scales, as an RCM is not intended to affect the large-scale flow (e.g. Giorgi and Mearns 1991; von Storch et al. 2000; Christensen et al. 2007). As a matter of a fact, large scales are sometimes misrepresented in RCM simulations and the application of largescale spectral nudging intends to prevent this from happening (e.g., von Storch et al. 2000; Biner et al. 
2000; Miguez-Macho et al. 2004; Castro et al. 2005). A contrarian contention has been upheld since a long time by some scientists such as Machenhauer (personal communication) and Mesinger (e.g. Mesinger et al. 2002; 2012), about the potential for reducing some aspects of large-scale errors present in LBC when using very large domain LAM. Our results, obtained within the idealised framework of the IBB, lend some support to this thesis in showing that RCM can have some, although limited, skill at improving imperfect large scales supplied as driving LBC. It is important to reiterate that, throughout this paper, the term "large scales" referred to those scales that are large but still resolved within the limited-area computational domain. Clearly it would be nonsensical to expect that a LAM could possibly improve upon planetary scales exceeding its computational domain, for phenomena such as El Niño-Southern Oscillation (ENSO) or North Atlantic Oscillation (NAO). As most RCM to this day are not coupled interactively to ocean models, prescribing sea surface temperature and sea-ice cover from GCM simulations also results in erroneous surface forcings in RCM simulations.

Finally, it is important to reiterate that small scales constitute the main expected added value of RCM simulations. As was to be expected, we found that small scales are indeed better simulated by high-resolution RCM than by coarser resolution driving model. On the other hand, while large-scale LBC errors eventually limit the skill of RCM at simulating adequately small scales, for intermediate-resolution driving model, the LB small-scale errors were found to be rather insensitive to LBC errors as well as to domain size.

Frequency distributions of simulated variables is also an aspect where there are expectations for added value of high-resolution RCM simulations. Indeed we found that RCM simulations improved markedly the precipitation distributions, independently of their domain size and despite LBC errors.

Acknowledgments This study was funded by the Québec's Ministère du Développement Économique, Innovation et Exportation (MDEIE), Hydro-Québec, the Ouranos Consortium on Regional Climatology and Adaptation to Climate Change, and the Natural Sciences and Engineering Research Council of Canada (NSERC). The calculations were made possible through an award from the Canadian Foundation for Innovation (CFI) to the Canada Research Chair for Regional Climate Modelling at UQAM. We would like to thank Mr Georges Huard and Mrs Nadjet Labassi for maintaining an efficient and user-friendly local computing facility, and Ms Katja Winger for helping with the GEMCLIM and CRCM5 simulations.

Open Access This article is distributed under the terms of the Creative Commons Attribution License which permits any use, distribution, and reproduction in any medium, provided the original author(s) and the source are credited.

\section{References}

Alexandru A, de Elía R, Laprise R (2007) Internal variability in regional climate downscaling at the seasonal scale. Mon Weather Rev 135(9):3221-3238

Antic S, Laprise R, Denis B, de Elía R (2004) Testing the downscaling ability of a one-way nested regional climate model in regions of complex topography. Clim Dyn 23:473-493

Arakawa A, Lamb VR (1977) Computational design of the basic dynamical process of the UCLA general circulation model. In: Methods in computational physics, vol 17. Academic Press, pp 173-265

Bélair S, Mailhot J, Girard C, Vaillancourt P (2005) Boundary layer and shallow cumulus clouds in a medium-range forecast of a large-scale weather system. Mon Weather Rev 133:1938-1959

Biner S, Caya D, Laprise R, Spacek S (2000) Nesting of RCMs by imposing large scales. In: Research activities in atmospheric and oceanic modelling, WMO/TD 987(30):7.3-7.4

Boer GJ (1982) Diagnostic equations in isobaric coordinates. Mon Weather Rev 110:1801-1820

Bukovsky MS (2011) Masks for the Bukovsky regionalization of North America, Regional Integrated Sciences Collective, Institute for Mathematics Applied to Geosciences, National Center for Atmospheric Research, Boulder, CO. Downloaded 2012-0618. http://www.narccap.ucar.edu/contrib/bukovsky/

Castro CL, Pielke RA, Leoncini G (2005) Dynamical downscaling: an assessment of value added using a regional climate model. J Geophys Res (Atmos) 110:D05108. doi:10.1029/2004JD004721

Christensen JH, Machenhauer B, Jones RG, Schär C, Ruti PM, Castro M, Visconti G (1997) Validation of present-day regional climate simulations over Europe: LAM simulations with observed boundary conditions. Clim Dyn 13:489-506

Christensen OB, Gaertner MA, Prego JA, Polcher J (2001) Internal variability of regional climate models. Clim Dyn 17:875-887

Christensen JH, Hewitson B, Busuioc A, Chen A, Gao X, Held I, Jones R, Kolli RK, Kwon W-T, Laprise R, Magaña Rueda V, Mearns L, Menéndez CG, Räisänen J, Rinke A, Sarr A, Whetton P (2007) Regional climate projections. In: Solomon S, Qin D, Manning M, Chen Z, Marquis M, Averyt KB, Tignor M, Miller HL (eds) Chap. 11 in Climate change 2007: the physical science basis. Contribution of working group I (WGI) to the fourth assessment report (AR4) of the intergovernmental panel on climate change (IPCC). Cambridge University Press, Cambridge. http://www.ipcc. ch/publications_and_data/ar4/wg1/en/ch11.html

Côté J, Gravel S, Méthot A, Patoine A, Roch M, Staniforth A (1998) The operational CMC-MRB global environmental multiscale (GEM) model. Part I: design considerations and formulation. Mon Weather Rev 126:1373-1395

Davies HC (1976) A lateral boundary formulation for multi-level prediction models. Q J R Meteorol Soc 102:405-418

Denis B, Côté J, Laprise R (2002a) Spectral decomposition of twodimensional atmospheric fields on limited-area domains using discrete cosine transforms (DCT). Mon Weather Rev 130: 1812-1829

Denis B, Laprise R, Caya D, Côté J (2002b) Downscaling ability of one-way-nested regional climate models: the Big-Brother experiment. Clim Dyn 18:627-646

Denis B, Laprise R, Caya D (2003) Sensitivity of a regional climate model to the spatial resolution and temporal updating frequency of the lateral boundary conditions. Clim Dyn 20:107-126

Diaconescu EP, Laprise R, Sushama L (2007) The impact of lateral boundary data errors on the simulated climate of a nested regional climate model. Clim Dyn 28(4):333-350

Diaconescu EP, Laprise R, Zadra A (2012) Singular vector decomposition of the internal variability of the Canadian regional 
climate. Clim Dyn 38(5-6):1093-1113. doi:10.1007/s00382011-1179-x

Dimitrijevic M, Laprise R (2005) Validation of the nesting technique in a RCM and sensitivity tests to the resolution of the lateral boundary conditions during summer. Clim Dyn 25:555-580

Frei C, Christensen JH, Déqué M, Jacob D, Jones RG, Vidale PL (2003) Daily precipitation statistics in regional climate models: evaluation and intercomparison for the European Alps. J Geophys Res 108(D3):4124. doi:10.1029/2002JD002287

Giorgi F, Bi X (2000) A study of internal variability of regional climate model. J Geophys Res 105:29503-29521

Giorgi F, Mearns LO (1991) Approaches to the simulation of regional climate change: a review. Rev Geophys 29:191-216

Gleckler P (1996) AMIP II guidelines. AMIP Newsletter, no. 8, PCMDI/LLNL. http://www-pcmdi.llnl.gov/projects/amip/NEWS

Herceg D, Sobel AH, Sun L, Zebiak SE (2006) The big brother experiment and seasonal predictability in the NCEP regional spectral model. Clim Dyn 26(4):1-14

Jacob D, Bärring L, Christensen OB, Christensen JH, de Castro M et al (2007) An intercomparison of regional climate models for Europe: model performance in present-day climate. Clim Change 81(Supplement 1):31-52

Jones RG, Murphy JM, Noguer M (1995) Simulation of climate change over Europe using a nested regional-climate model. I: assessment of control climate, including sensitivity to location of lateral boundaries. Q J R Meteorol Soc 121:1413-1449

Kain JS, Fritsch JM (1990) A one-dimensional entraining/detraining plume model and application in convective parameterization. J Atmos Sci 47:2784-2802

Køltzow M, Iversen T, Haugen JE (2008) Extended big-brother experiments: the role of lateral boundary data quality and size of integration domain in regional climate modelling. Tellus A 60(3):398-410

Kuo HL (1965) On formation and intensification of tropical cyclones through latent heat release by cumulus convection. J Atmos Sci 22:4063

Laprise R (1992) The Euler equations of motion with hydrostatic pressure as an independent variable. Mon Weather Rev 120: 197-207

Laprise R, de Elía R, Caya D, Biner S, Lucas-Picher P, Diaconescu EP, Leduc M, Alexandru A, Šeparović L (2008) Challenging some tenets of regional climate modelling. Meteorol Atmos Phys Spec Issue Reg Clime Stud 100:3-22

Laprise R, Kornic D, Rapaic M, Separovic L, Leduc M, Nikiema O, Di Luca A, Diaconescu E, Alexandru A, Lucas-Picher P, de Elía R, Caya D, Biner S (2012) Considerations of domain size and large-scale driving for nested regional climate models: impact on internal variability and ability at developing small-scale details. In: Berger A, Mesinger F, Šijači D (eds) Climate change: inferences from Paleoclimate and regional aspects. Springer, Part 4, 201-214. doi:10.1007/978-3-7091-0973-1_15

Leduc M, Laprise R (2009) Regional climate model sensitivity domain size. Clim Dyn 32(6):833-854

Leduc M, Laprise R, Moretti-Poisson M, Morin J-P (2011) Sensitivity to domain size of mid-latitude summer simulations with a regional climate model. Clim Dyn 37:343-356. doi:10.1007/ s00382-011-1008-2

Li J, Barker HW (2005) A radiation algorithm with correlated-k distribution. Part I: local thermal equilibrium. J Atmos Sci 62:286-309

Liang XZ, Wang WC (1995) A GCM study of the climatic effect of 1979-1992 ozone trend. In: Wang WC, Isaksen ISA (eds) Atmospheric ozone as a climate gas. NATO ASI series, pp 259-288

Lucas-Picher P, Caya D, de Elía R, Laprise R (2008a) Investigation of regional climate models' internal variability with a ten-member ensemble of ten-year simulations over a large domain. Clim Dyn 31(7-8):927-940

Lucas-Picher P, Caya D, Biner S, Laprise R (2008b) Quantification of the lateral boundary forcing in a regional climate model using an ageing tracer. Mon Weather Rev 136:4980-4996. doi:10.1175/ 2008MWR2448.1

Machenhauer B, Windelband M, Botzet M, Hesselbjerg J, Déqué M, Jones GR, Ruti PM, Visconti G (1998) Validation and analysis of regional present-day climate and climate change simulations over Europe, Max-Planck Institute of Meteorology Hamburg, report no. $275,87 \mathrm{pp}$

McFarlane NA (1987) The effect of orographically excited gravitywave drag on the circulation of the lower stratosphere and troposphere. J Atmos Sci 44:1175-1800

Mesinger F, Brill K, Chuang H, DiMego G, Rogers E (2002) Limited area predictability: can upscaling also take place? Res Act Atmos Ocean Mod, rep 32, WMO/TD no 1105, 5.30-5.31

Mesinger F, Veljovic K, Fennessy MJ, Altshuler EL (2012) Value added in regional climate modeling: should one aim to improve on the large scales as well? In: Berger A, Mesinger F, Śijači D (eds) Climate change: inferences from Paleoclimate and regional aspects. Springer, Part 4, 201-214. doi:10.1007/978-3-70910973-1_15

Miguez-Macho G, Stenchikov GL, Robock A (2004) Spectral nudging to eliminate the effects of domain position and geometry in regional climate model simulations. J Geophys Res 109:D13104. doi:10.1029/2003JD004495

Misra V (2007) Addressing the issue of systematic errors in a regional climate model. J Climate 20:801-818

Nikiéma O, Laprise R (2011) Budget study of the internal variability in ensemble simulations of the Canadian RCM at the seasonal scale. J Geophys Res Atmos 116:D16112. doi:10.1029/2011JD015841

Noguer M, Jones R, Murphy J (1998) Sources of systematic errors in the climatology of a regional climate model over Europe. Clim Dyn 14:691-712

Radu R, Déqué M, Somot S (2008) Spectral nudging in a spectral regional climate model. Tellus A 60:898-910. doi:10.1111/ j.1600-0870.2008.00341.x

Rapaic M, Leduc M, Laprise R (2011) Evaluation of the internal variability and estimation of the downscaling ability of the Canadian regional climate model for different domain sizes over the north Atlantic region using the Big-Brother experimental approach. Clim Dyn 36(9-10):1979-2001

Rinke A, Dethloff K (2000) On the sensitivity of a regional Arctic climate model to initial and boundary conditions. Clim Res 14: 101-113

Rinke A, Marbaix P, Dethloff K (2004) Internal variability in Arctic regional climate simulations: case study for the Sheba year. Clim Res 27:197-209

Rinke A, Dethloff K, Cassano JJ, Christensen JH, Curry JA, Du P, Girard E, Haugen J-E, Jacob D, Jones CG, Køltzow M, Laprise R, Lynch AH, Pfeifer S, Serreze MC, Shaw MJ, Tjernström M, Wyser K, Zagar M (2006) Evaluation of an ensemble of Arctic regional climate models: spatiotemporal fields during the SHEBA year. Clim Dyn 26(5):459-472. doi:10.1007/s00382005-0095-3

Robert A, Yakimiw E (1986) Identification and elimination of an inflow boundary computational solution in limited area model integrations. Atmos Ocean 24:369-385

Rummukainen M (2010) State-of-the-art with regional climate models. WIREs Clim Change 1:82-96. doi:10.1002/wcc.8

Sundqvist H, Berge E, Kristjansson JE (1989) Condensation and cloud parameterization studies with a mesoscale numerical weather prediction model. Mon Weather Rev 117:1641-1657

Taylor KE (2001) Summarizing multiple aspects of model performance in a single diagram. J Geophys Res 106(D7):7183-7192 
Veljovic K, Rajkovic B, Fennessy MJ, Altshuler EL, Mesinger F (2010) Regional climate modeling: should one attempt improving on the large scales? Lateral boundary condition scheme: any impact? Meteorol Z 19(3):237-246

Verseghy DL (2000) The Canadian Land Surface Scheme (CLASS): its history and future. Atmos Ocean 38(1):1-13

Verseghy DL, McFarlane NA, Lazare M (1993) CLASS-A Canadian land surface scheme for GCMS II. Vegetation model and coupled runs. Int J Climatol 13(4):347-370

von Storch H, Langenberg H, Feser F (2000) A spectral nudging technique for dynamical downscaling purposes. Mon Weather Rev 128:3664-3673

Yakimiw E, Robert A (1990) Validation experiments for nested gridpoint regional forecast model. Atmos Ocean 28:466-472
Yang H, Wang B, Wang B (2011) Reduction of systematic biases in regional climate downscaling through ensemble forcing. Clim Dyn 38(3-4):655-665

Yeh KS, Côté J, Gravel S, Methot A, Patoine A, Roch M, Staniforth A (2002) The operational CMC-MRB global environmental multiscale (GEM) model. Part III: non-hydrostatic formulation. Mon Weather Rev 130:339-356

Zadra A, Roch M, Laroche S, Charron M (2003) The subgrid scale orographic blocking parameterization of the GEM model. Atmos Ocean 41:155-170

Zadra A, Caya D, Côté J, Dugas B, Jones C, Laprise R, Winger K, Caron LP (2008) The next Canadian regional climate model. Phys Canada 64(2):75-83 